\title{
Ancient genomes show social and reproductive behavior of early Upper Paleolithic foragers
}

Sikora, Martin; Seguin-Orlando, Andaine; Sousa, Vitor C.; Albrechtsen, Anders; Korneliussen, Thorfinn; Ko, Amy; Rasmussen, Simon; Dupanloup, Isabelle; Nigst, Philip R.; Bosch, Marjolein D.

Total number of authors:

27

Published in:

Science

Link to article, DOI:

10.1126/science.aao1807

Publication date:

2017

Document Version

Peer reviewed version

Link back to DTU Orbit

Citation (APA):

Sikora, M., Seguin-Orlando, A., Sousa, V. C., Albrechtsen, A., Korneliussen, T., Ko, A., Rasmussen, S., Dupanloup, I., Nigst, P. R., Bosch, M. D., Renaud, G., Allentoft, M. E., Margaryan, A., Vasilyev, S. V.

Veselovskaya, E. V., Borutskaya, S. B., Deviese, T., Comeskey, D., Higham, T., ... Willerslev, E. (2017). Ancient genomes show social and reproductive behavior of early Upper Paleolithic foragers. Science, 358(6363), 659662. [eaao1807]. https://doi.org/10.1126/science.aao1807

\section{General rights}

Copyright and moral rights for the publications made accessible in the public portal are retained by the authors and/or other copyright owners and it is a condition of accessing publications that users recognise and abide by the legal requirements associated with these rights.

- Users may download and print one copy of any publication from the public portal for the purpose of private study or research.

- You may not further distribute the material or use it for any profit-making activity or commercial gain

- You may freely distribute the URL identifying the publication in the public portal 


\title{
Ancient genomes show social and reproductive behavior of early Upper Paleolithic foragers
}

\author{
Martin Sikora, ${ }^{1 *}$ Andaine Seguin-Orlando, ${ }^{1 *}$ Vitor C. Sousa, ${ }^{2,3,4}$ Anders Albrechtsen, ${ }^{5}$ Thorfinn \\ Korneliussen, ${ }^{1}$ Amy Ko, ${ }^{6}$ Simon Rasmussen, ${ }^{7}$ Isabelle Dupanloup,,${ }^{2,3}$ Philip R. Nigst, ${ }^{8}$ Marjolein D. \\ Bosch, ${ }^{9,10}$ Gabriel Renaud, ${ }^{1}$ Morten E. Allentoft, ${ }^{1}$ Ashot Margaryan, ${ }^{1,11}$ Sergey V. Vasilyev, ${ }^{12}$ Elizaveta V. \\ Veselovskaya, ${ }^{12}$ Svetlana B. Borutskaya, ${ }^{13}$ Thibaut Deviese, ${ }^{14}$ Dan Comeskey, ${ }^{14}$ Tom Higham, ${ }^{14}$ Andrea \\ Manica, ${ }^{15}$ Robert Foley, ${ }^{1,16}$ David J. Meltzer, ${ }^{1,17}$ Rasmus Nielsen, ${ }^{1,5}$ Laurent Excoffier, ${ }^{2,3}$ Marta Mirazon \\ Lahr, ${ }^{1,16}$ Ludovic Orlando, ${ }^{1,18}$ Eske Willerslev ${ }^{1,19,20} \uparrow$
}

${ }^{1}$ Centre for GeoGenetics, Natural History Museum of Denmark, University of Copenhagen, 1350 Copenhagen, Denmark. ${ }^{2}$ Institute of Ecology and Evolution, University of Bern, CH-3012 Bern, Switzerland. ${ }^{3}$ Swiss Institute of Bioinformatics, 1015 Lausanne, Switzerland. ${ }^{4}$ Centre for Ecology, Evolution and Environmental Changes, Faculdade de Ciências, Universidade de Lisboa, 1749-016 Lisboa, Portugal. ${ }^{5}$ Department of Biology, University of Copenhagen, 2200 København N, Denmark. ${ }^{6}$ Department of Integrative Biology, University of California, Berkeley, CA 94720, USA. ${ }^{7}$ Center for Biological Sequence Analysis, Department of Systems Biology, Technical University of Denmark, Kemitorvet, 2800 Kongens Lyngby, Denmark. ${ }^{8}$ Division of Archaeology, Department of Archaeology and Anthropology, University of Cambridge, Cambridge CB2 3DZ, UK. ${ }^{9} \mathrm{McD}$ onald Institute for Archaeological Research, University of Cambridge, Cambridge CB2 3ER, UK. ${ }^{10}$ Department of Human Evolution, Max Planck Institute for Evolutionary Anthropology, 04103 Leipzig, Germany. ${ }^{11}$ nstitute of Molecular Biology, National Academy of Sciences, 0014 Yerevan, Armenia. ${ }^{12}$ Centre of Physical Anthropology, Institute Ethnology and Anthropology, Russian Academy of Science, 119334 Moscow, Russia. ${ }^{13}$ Department of Anthropology, Biological Faculty, Lomonosov's Moscow State University, 119991 Moscow, Russia. ${ }^{14}$ Oxford Radiocarbon Accelerator Unit, University of Oxford, Oxford OX1 3QY, UK. ${ }^{15}$ Department of Zoology, University of Cambridge, CB2 3EJ, UK. ${ }^{16}$ Leverhulme Centre for Human Evolutionary Studies, Department of Archaeology and Anthropology, University of Cambridge, Cambridge CB2 1QH, UK. ${ }^{17}$ Department of Anthropology, Southern Methodist University, Dallas, TX 75275, USA. ${ }^{18}$ Laboratoire d'Anthropobiologie Moléculaire et d'Imagerie de Synthèse, CNRS UMR 5288, Université de Toulouse, Université Paul Sabatier, 31000 Toulouse, France. ${ }^{19}$ GeoGenetics Groups, Department of Zoology, University of Cambridge, Cambridge CB2 3EJ, UK. ${ }^{20}$ Wellcome Trust Sanger Institute, Hinxton, Cambridge CB10 1SA, UK.

*These authors contributed equally to this work.

†Corresponding author. Email: ewillerslev@snm.ku.dk

Present-day hunter-gatherers (HGs) live in multilevel social groups essential to sustain a population structure characterized by limited levels of within-band relatedness and inbreeding. When these wider social networks evolved among HGs is unknown. Here, we investigate whether the contemporary HG strategy was already present in the Upper Paleolithic (UP), using complete genome sequences from Sunghir, a site dated to $\sim 34$ thousand years BP (kya) containing multiple anatomically modern human $(A M H)$ individuals. We demonstrate that individuals at Sunghir derive from a population of small effective size, with limited kinship and levels of inbreeding similar to HG populations. Our findings suggest that UP social organization was similar to that of living HGs, with limited relatedness within residential groups embedded in a larger mating network.

Opportunities to investigate the population dynamics of early AMH populations are rare owing to a dearth of human remains, with wide variations in ancient population size estimates from ethnographic or archaeological data $(1,2)$. In the absence of evidence for true contemporaneity among individuals recovered archaeologically, the population structure of foraging groups is even harder to establish. Exceptions are cases of multiple UP individuals buried simultaneously or originating from sufficiently close temporal and spatial proximity that they may represent a single social group.

One such example of multiple burials is Sunghir, a site harboring two of the most extraordinary UP burials known $(3,4)$ (figs. S1 and S2 and tables S1 to S4): one of an adult male (Sunghir 1 [SI]), and another one of two sub-adults (Sunghir 2 and 3 [SII and SIII]), originally thought to be a boy and girl, interred head-to-head. All remains were covered in ochre, and accompanied by rich grave goods including ivory beads and spears, armbands and carvings, as well as arctic fox canines. Adjacent to SII was the femoral diaphysis of an adult (Sunghir 4 [SIV]) that had been polished, hollowed-out and filled with red ochre. The site also yielded other less complete human remains, some of uncertain stratigraphic provenance (Sunghir 5-9 [SV-SIX]). Radiocarbon analyses place the age of SI-SIV between 34.6 and 33.6 kya $(5,6)$. The homogeneity in morphological traits (e.g., metopism) among the remains, as well as signs of possible congenital pathologies in SIII have been interpreted as evidence of inbreeding (3). Other UP individuals with reported congenital or degenerative pathologies (e.g., at Barma Grande and Dolní Věstonice) (3) reinforce the view that UP groups were small and susceptible to inbreeding, possibly 
similar to what has been reported for the Altai Neanderthal (7). However, genomic data available for some of those individuals (8) were of insufficient coverage for inferring population sizes and inbreeding levels.

We screened six of the Sunghir individuals (SI-SV; Sunghir 6 [SVI]) to assess DNA preservation, five of which (excluding SV) yielded sufficient endogenous DNA for genome sequencing. We sequenced those genomes to an average depth-of-coverage ranging from 1.11X to 10.75X (figs. S3 and S4 and tables S5 and S6), and compared them to panels of modern and ancient human genomes (4). All individuals were genetically male on the basis of the fraction of Y chromosome reads (table S7), including SIII who was previously identified as female (3). Contamination levels from X chromosome heterozygosity were low $(0.33 \%-0.90 \%$, table S5) except for SVI (13.1\%). Radiocarbon dates indicate that while SV is only slightly more recent than the other individuals, SVI is from $\sim 900$ yBP (figs. S5 and S6 and tables S8 to S10). Together with mtDNA and Y-chromosome haplogroups (W3a1 and I2a1b2, respectively), these data indicate that SVI is not associated with the UP burials at the site, and was therefore excluded from further analyses.

Analyses of mtDNA genomes place SI-SIV in haplogroup U, consistent with West Eurasian and Siberian Paleolithic and Mesolithic genomes (9) (fig. S7 and table S5). SI belonged to haplogroup U8c; the sequences for the three individuals from the double burial (SII-SIV) were identical, and belonged to haplogroup U2, which is closely related to the UP Kostenki 12 (8) and Kostenki 14 (10) individuals. Phylogenetic analyses of the $\mathrm{Y}$ chromosome sequences place all Sunghir individuals in an early divergent lineage of haplogroup Cla2 (fig. S8 and tables S12 to S15). Y-chromosome haplogroup $\mathrm{Cl}$, which is rare among contemporary Eurasians, has been found in other early European individuals including the $\sim 36$ ky-old Kostenki 14 (11).

We investigated the degree of relatedness among the Sunghir individuals with a method that allows relationship inferences up to a third degree, but does not rely on allele frequencies (4). Surprisingly, none of them were closely related (that is, third degree or closer), despite the fact that the SII-SIV individuals buried together share both mitochondrial and Y-chromosome lineages (Fig. 1 and tables S16 to S23). We then inferred genomic segments that were identical-by-descent (IBD) and homozygous-by-descent (HBD) from three higher coverage Sunghir genomes (SII-SIV) and a panel of ancient and contemporary humans (4). We compared their distributions to those inferred from whole genomes obtained using coalescent simulations (12) of randomly mating populations with varying effective population sizes $\left(\mathrm{N}_{\mathrm{E}}\right)$ (fig. S9). The distribution of HBD tracts were different between AMH and archaic humans, indicating small effective population sizes and/or recent inbreeding in archaic individuals, particularly the Altai Neanderthal $(7,13)$ (Fig. 2A and figs. S10 to S14).

Patterns of pairwise IBD-sharing detect close genetic relatives in modern individuals (Fig. 2B). However, the Sunghir pairs do not share sufficiently long IBD tracts to suggest relatedness at the $1^{\text {st }}$ or $2^{\text {nd }}$ degree, consistent with the results from genome-wide identity-by-state (IBS) counts (Fig. 2B and fig. S15). Interestingly, the effective population sizes tended to be higher $\left(\mathrm{N}_{\mathrm{E}} \sim 500\right)$ for two out of three Sunghir pairs than those estimated from HBD segments $\left(\mathrm{N}_{\mathrm{E}}\right.$ $\sim 200$ ). $\mathrm{N}_{\mathrm{E}}$ from both HBD and IBD tracts (4) was within the range, or slightly higher, than that of contemporary nonAfrican HG populations (Fig. 2C), particularly from genetically isolated groups (14).

Genetic clustering of ancient individuals using outgroup $f_{3}$ statistics $f_{3}\left(\right.$ Mbuti; Ancient ${ }_{1}$, Ancient $\left.{ }_{2}\right)$ indicates shared genetic drift and tight clustering of the Sunghir individuals, which form a clade to the exclusion of all other individuals (Fig. 3, figs. S16 to S20, and tables S28 and S29). Furthermore, we find genetic affinities between the Sunghir individuals and those from Kostenki (12 and 14), as well as with the 'Vêstonice cluster' (8), associated with the UP Gravettian culture.

Individuals mapped onto a previously inferred admixture graph of early Eurasians $(4,8)$ placed the Sunghir cluster as a descendent of a lineage related to the Kostenki 14 individual, contributing the major fraction of the ancestry of the 'Vêstonice cluster' (Fig. 3C and figs. S21 to S24). Adding the low coverage Kostenki 12 individual suggests a closer relationship to the Sunghir group rather than with the earlier Kostenki 14 individual (fig. S25). Finally, Kostenki 14 shows substantial population-specific drift after its divergence from the shared ancestor with Sunghir, allowing us to reject a direct ancestral relationship to both Sunghir and Kostenki 12 (fig. S26). These results suggest that the people at Kostenki were at least partially replaced by later groups related to Sunghir, which exhibit genetic affinities with individuals of the more western Gravettian culture.

Our high coverage Sunghir individual (SIII), allows us to explore quantitative models of Eurasian demographic history. Using coalescent-based modeling of the site frequency spectrum (SFS) (fig. S27), we estimate that SIII diverged $\sim 38$ kya (95\% CI $35-43)$ from the lineage ancestral to contemporary Europeans, with a relatively small effective population size $\left(\mathrm{N}_{\mathrm{e}}=297\right.$; $95 \%$ CI 158-901) (Fig. 4A, figs. S28 and S29, and tables S24 and S25). The Ust'-Ishim genome, a 45 kya UP individual from Siberia (15) which diverged from the Asian lineage ( $48 \mathrm{kya} ; 95 \%$ CI $45-55)$ soon after the initial divergence among Eurasians ( 52.5 kya; $95 \%$ CI 49 57 ), indicates a comparably higher effective population size $(\mathrm{Ne}=1,203$; 95\% CI 253-7098) (Fig. 4B, figs. S30 and S31, and tables S26 and S27). The best-fit models also suggest a 
common Neanderthal admixture event shared by all Eurasians at 55 kya (95\% CI 52-63), consistent with previous estimates $(11,15)$. However, we also find evidence of multiple Neanderthal admixture events in both SIII (36 kya; 95\% CI 34-42) and Ust'-Ishim (47 kya; 95\% CI 44-51), the latter introgression providing an estimated $0.6 \%$ (95\% CI 0.002-1.53) of Neanderthal ancestry to SIII. That excess may reflect either further pulses of Neanderthal introgression, or selection against Neanderthal introgressed regions in AMHs as previously suggested $(8,16-18)$. Analyses of putative archaicintrogressed genomic segments (4) confirms a higher level of Neanderthal ancestry and a longer average Neanderthal segment length among UP individuals, in agreement with their closer proximity to the human-Neanderthal admixture event than present-day Eurasians (fig. S36). Assuming that the Sunghir individuals are contemporaneous (4), we obtain a refined estimate of the time since admixture at 770 generations (95\% CI 755-786). Accounting for the uncertainty of both the admixture estimate and ${ }^{14} \mathrm{C}$ ages, this corresponds to an admixture date between the ancestors of Sunghir and Neanderthals between 53.6 and 58.1 kya (at 29 years/generation (19)), in agreement with the results obtained from coalescent modeling (fig. S37).

Our results suggest a social and population network of HG demes that preferentially mated within sub-groups, with exogamy and regular exchanges between demes. Among contemporary HGs, primary kin constitute $<10 \%$ of residential groups, leading to low genetic relatedness $(20,21)$. Some modern human groups exhibit increased levels of inbreeding, including populations where consanguineous marriage practices are encouraged, or geographically isolated HG groups such as those from the Amazon rainforest region (Fig. 2, A and C). In contrast, patterns of HBD among the UP individuals are consistent with randomly mating populations of moderate effective size $\left(\mathrm{N}_{\mathrm{E}} \sim 200\right)$, suggesting that close consanguineous mating was avoided (Fig. 2, A and C). Although our findings are currently limited to a single UP site, if they are representative of early UP HGs more generally, they reveal a social structure and cultural practices that emphasized exogamy. This is consistent with archaeological evidence of high mobility in the UP (22), perhaps comparable to the scale of mobility seen ethnographically among small foraging bands at high-latitudes (23). We note that this interpretation relies on the evidence that all individuals at Sunghir were contemporaneous and members of the same social group. While this is clearly the case for the two children in the double burial (SII, SIII), it is possible that both SIV and SI were members of different social groups, potentially separated in time from SII and SIII. Nevertheless, the shared material culture, overlapping radiocarbon date intervals, as well as close genetic relationship among all individuals, all support this inference.
Although the number of ancient genomes available remains small, the differences in inbreeding levels, and thus group organization, between AMH groups in the UP and Neanderthals are intriguing. The small reproductive groups of UP AMH at Sunghir apparently avoided inbreeding and its deleterious consequences, in contrast to what has been observed for the Altai Neanderthals. We caution that more genomic data on Neanderthals from other regions is necessary to conclude whether the patterns observed in the Altai are representative of their genetic diversity more generally, or if that individual was an outlier. Assuming the former, whether this would reflect ongoing extinction of Neanderthals or a more general difference in social behavior and cultural practices also remains unknown. Our results nonetheless suggest that the human HG social structure of low levels of within-band relatedness, complex family residence patterns, relatively high individual mobility and multilevel social networks were already in place among UP societies 34 kya. This social structure may have affected the development of cooperation and information transfer that underlie the evolution of culture in humans $(20,21,24,25)$, and may be crucial to understanding our species unique evolutionary resilience and trajectory.

\section{REFERENCES AND NOTES}

1. J.-P. Bocquet-Appel, P.-Y. Demars, L. Noiret, D. Dobrowsky, Estimates of Upper Palaeolithic meta-population size in Europe from archaeological data. J. Archaeol. Sci. 32, 1656-1668 (2005). doi:10.1016/j.jas.2005.05.006

2. M. M. Lahr, R. A. Foley, in Neanderthals and Modern Humans in the European Landscape During the Last Glaciation: Archaeological Results of the Stage 3 Project (McDonald Institute for Archaeological Research, Cambridge, 2003), pp. 241-256.

3. E. Trinkaus, A. P. Buzhilova, M. B. Mednikova, M. V. Dobrovolskaya, The People of Sunghir: Burials, Bodies, and Behavior in the Earlier Upper Paleolithic (Oxford Univ. Press, 2014)

4. See supplementary materials.

5. A. Marom, J. S. O. McCullagh, T. F. G. Higham, A. A. Sinitsyn, R. E. M. Hedges, Single amino acid radiocarbon dating of Upper Paleolithic modern humans. Proc. Natl. Acad. Sci. U.S.A. 109, 6878-6881 (2012). doi:10.1073/pnas.1116328109 Medline

6. S. Nalawade-Chavan, J. McCullagh, R. Hedges, New hydroxyproline radiocarbon dates from Sungir, Russia, confirm early Mid Upper Palaeolithic burials in Eurasia. PLOS ONE 9, e76896 (2014). doi:10.1371/journal.pone.0076896 Medline

7. K. Prüfer, F. Racimo, N. Patterson, F. Jay, S. Sankararaman, S. Sawyer, A. Heinze, G. Renaud, P. H. Sudmant, C. de Filippo, H. Li, S. Mallick, M. Dannemann, Q. Fu, M. Kircher, M. Kuhlwilm, M. Lachmann, M. Meyer, M. Ongyerth, M. Siebauer, C. Theunert, A. Tandon, P. Moorjani, J. Pickrell, J. C. Mullikin, S. H. Vohr, R. E. Green, I. Hellmann, P. L. F. Johnson, H. Blanche, H. Cann, J. O. Kitzman, J. Shendure, E. E. Eichler, E. S. Lein, T. E. Bakken, L. V. Golovanova, V. B. Doronichev, M. V. Shunkov, A. P. Derevianko, B. Viola, M. Slatkin, D. Reich, J. Kelso, S. Pääbo, The complete genome sequence of a Neanderthal from the Altai Mountains. Nature 505, 43-49 (2014). doi:10.1038/nature12886 Medline

8. Q. Fu, C. Posth, M. Hajdinjak, M. Petr, S. Mallick, D. Fernandes, A. Furtwängler, W. 
Haak, M. Meyer, A. Mittnik, B. Nickel, A. Peltzer, N. Rohland, V. Slon, S. Talamo, I. Lazaridis, M. Lipson, I. Mathieson, S. Schiffels, P. Skoglund, A. P. Derevianko, N. Drozdov, V. Slavinsky, A. Tsybankov, R. G. Cremonesi, F. Mallegni, B. Gély, E. Vacca, M. R. Morales, L. G. Straus, C. Neugebauer-Maresch, M. Teschler-Nicola, S. Constantin, O. T. Moldovan, S. Benazzi, M. Peresani, D. Coppola, M. Lari, S. Ricci, A. Ronchitelli, F. Valentin, C. Thevenet, K. Wehrberger, D. Grigorescu, H. Rougier, I. Crevecoeur, D. Flas, P. Semal, M. A. Mannino, C. Cupillard, H. Bocherens, N. J. Conard, K. Harvati, V. Moiseyev, D. G. Drucker, J. Svoboda, M. P. Richards, D. Caramelli, R. Pinhasi, J. Kelso, N. Patterson, J. Krause, S. Pääbo, D. Reich, The genetic history of Ice Age Europe. Nature 534, 200-205 (2016). doi:10.1038/nature17993 Medline

9. C. Posth, G. Renaud, A. Mittnik, D. G. Drucker, H. Rougier, C. Cupillard, F. Valentin, C. Thevenet, A. Furtwängler, C. Wißing, M. Francken, M. Malina, M. Bolus, M. Lari, E. Gigli, G. Capecchi, I. Crevecoeur, C. Beauval, D. Flas, M. Germonpré, J. van der Plicht, R. Cottiaux, B. Gély, A. Ronchitelli, K. Wehrberger, D. Grigorescu, J. Svoboda, P. Semal, D. Caramelli, H. Bocherens, K. Harvati, N. J. Conard, W. Haak, A. Powell, J. Krause, Pleistocene mitochondrial genomes suggest a single major dispersal of non-Africans and a late glacial population turnover in Europe. Curr. Biol. 26, 827-833 (2016). doi:10.1016/i.cub.2016.02.022 Medline

10. J. Krause, A. W. Briggs, M. Kircher, T. Maricic, N. Zwyns, A. Derevianko, S. Pääbo, A complete mtDNA genome of an early modern human from Kostenki, Russia. Curr. Biol. 20, 231-236 (2010). doi:10.1016/j.cub.2009.11.068 Medline

11. A. Seguin-Orlando, T. S. Korneliussen, M. Sikora, A.-S. Malaspinas, A. Manica, I. Moltke, A. Albrechtsen, A. Ko, A. Margaryan, V. Moiseyev, T. Goebel, M. Westaway, D. Lambert, V. Khartanovich, J. D. Wall, P. R. Nigst, R. A. Foley, M. M. Lahr, R. Nielsen, L. Orlando, E. Willerslev, Genomic structure in Europeans dating back at least 36,200 years. Science 346, 1113-1118 (2014). doi:10.1126/science.aaa0114 Medline

12. J. Kelleher, A. M. Etheridge, G. McVean, Efficient coalescent simulation and genealogical analysis for large sample sizes. PLOS Comput. Biol. 12, e1004842 (2016). doi:10.1371/journal.pcbi.1004842 Medline

13. M. Meyer, M. Kircher, M.-T. Gansauge, H. Li, F. Racimo, S. Mallick, J. G. Schraiber, F. Jay, K. Prüfer, C. de Filippo, P. H. Sudmant, C. Alkan, Q. Fu, R. Do, N. Rohland, A. Tandon, M. Siebauer, R. E. Green, K. Bryc, A. W. Briggs, U. Stenzel, J. Dabney, J. Shendure, J. Kitzman, M. F. Hammer, M. V. Shunkov, A. P. Derevianko, N. Patterson, A. M. Andrés, E. E. Eichler, M. Slatkin, D. Reich, J. Kelso, S. Pääbo, A high-coverage genome sequence from an archaic Denisovan individual. Science 338, 222-226 (2012). doi:10.1126/science.1224344 Medline

14. M. Kirin, R. McQuillan, C. S. Franklin, H. Campbell, P. M. McKeigue, J. F. Wilson, Genomic runs of homozygosity record population history and consanguinity. PLOS ONE 5, e13996 (2010). doi:10.1371/journal.pone.0013996 Medline

15. Q. Fu, H. Li, P. Moorjani, F. Jay, S. M. Slepchenko, A. A. Bondarev, P. L. F. Johnson, A. Aximu-Petri, K. Prüfer, C. de Filippo, M. Meyer, N. Zwyns, D. C. Salazar-García, Y. V. Kuzmin, S. G. Keates, P. A. Kosintsev, D. I. Razhev, M. P. Richards, N. V. Peristov, M. Lachmann, K. Douka, T. F. G. Higham, M. Slatkin, J.-J. Hublin, D. Reich, J. Kelso, T. B. Viola, S. Pääbo, Genome sequence of a 45,000year-old modern human from western Siberia. Nature 514, 445-449 (2014). doi:10.1038/nature13810 Medline

16. B. Vernot, J. M. Akey, Resurrecting surviving Neandertal lineages from modern human genomes. Science 343, 1017-1021 (2014). doi:10.1126/science.1245938 Medline

17. I. Juric, S. Aeschbacher, G. Coop, The strength of selection against Neanderthal introgression. PLOS Genet. 12, e1006340 (2016). doi:10.1371/journal.pgen.1006340 Medline

18. K. Harris, R. Nielsen, The genetic cost of Neanderthal introgression. Genetics 203, 881-891 (2016). doi:10.1534/genetics.116.186890 Medline

19. J. N. Fenner, Cross-cultural estimation of the human generation interval for use in genetics-based population divergence studies. Am. J. Phys. Anthropol. 128,
415-423 (2005). doi:10.1002/ajpa.20188 Medline

20. K. R. Hill, R. S. Walker, M. Bozicević, J. Eder, T. Headland, B. Hewlett, A. M. Hurtado, F. Marlowe, P. Wiessner, B. Wood, Co-residence patterns in huntergatherer societies show unique human social structure. Science 331, 1286-1289 (2011). doi:10.1126/science.1199071 Medline

21. M. Dyble, G. D. Salali, N. Chaudhary, A. Page, D. Smith, J. Thompson, L. Vinicius, R. Mace, A. B. Migliano, Sex equality can explain the unique social structure of hunter-gatherer bands. Science 348, 796-798 (2015). doi:10.1126/science.aaa5139 Medline

22. H. Floss, Rohmaterialversorgung im Paläolithikum des Mittelrheingebietes Archäol. Inform. 14, 113-117 (1991). doi:10.11588/ai.1991.1.21379

23. R. L. Kelly, The Lifeways of Hunter-Gatherers: The Foraging Spectrum (Cambridge Univ. Press, 2013).

24. R. Boyd, R. H. Schonmann, R. Vicente, Hunter-gatherer population structure and the evolution of contingent cooperation. Evol. Hum. Behav. 35, 219-227 (2014). doi:10.1016/i.evolhumbehav.2014.02.002

25. A. B. Migliano, A. E. Page, J. Gómez-Gardeñes, G. D. Salali, S. Viguier, M. Dyble, J. Thompson, N. Chaudhary, D. Smith, J. Strods, R. Mace, M. G. Thomas, V. Latora, L. Vinicius, Characterization of hunter-gatherer networks and implications for cumulative culture. Nat. Hum. Behav. 1, 0043 (2017). doi:10.1038/s41562-0160043

26. N. O. Bader, Sunghir': An Upper Palaeolithic Site (Nauka, 1978). [In Russian]

27. T. I. Alexeeva, N. O. Bader, Eds., Homo sungirensis. Upper Palaeolithic Man: Ecological and Evolutionary Aspects of the Investigation (Scientific World, Moscow, 2000).

28. O. N. Bader, N. O. Bader, in Homo sungirensis. Upper Palaeolithic Man: Ecological and Evolutionary Aspects of the Investigation, T. I. Alexeeva, N. O. Bader, Eds. (Scientific World, Moscow, 2000), pp. 21-29.

29. P. B. Pettitt, N. O. Bader, Direct AMS radiocarbon dates for the Sungir mid Upper Palaeolithic burials. Antiquity 74, 269-270 (2000). doi:10.1017/S0003598X00059196

30. A. A. Zubov, V. M. Kharitonov, Sungir Anthropological Investigations (Scientific World, Moscow, 1984)

31. N. O. Bader, Upper Palaeolithic Site Sungir (Graves and Environment) (Scientific World, Moscow, 1998).

32. P. J. Reimer, E. Bard, A. Bayliss, J. W. Beck, P. G. Blackwell, C. B. Ramsey, C. E. Buck, H. Cheng, R. L. Edwards, M. Friedrich, P. M. Grootes, T. P. Guilderson, H. Haflidason, I. Hajdas, C. Hatté, T. J. Heaton, D. L. Hoffmann, A. G. Hogg, K. A. Hughen, K. F. Kaiser, B. Kromer, S. W. Manning, M. Niu, R. W. Reimer, D. A. Richards, E. M. Scott, J. R. Southon, R. A. Staff, C. S. M. Turney, J. van der Plicht, IntCal13 and Marine13 radiocarbon age calibration curves $0-50,000$ years cal BP. Radiocarbon 55, 1869-1887 (2013). doi:10.2458/azu_js_rc.55.16947

33. C. B. Ramsey, Bayesian analysis of radiocarbon dates. Radiocarbon $51,337-360$ (2009). doi:10.1017/s0033822200033865

34. A. P. Buzhilova, The environment and health condition of the upper palaeolithic sunghir people of Russia. J. Physiol. Anthropol. Appl. Human Sci. 24, 413-418 (2005). doi:10.2114/jpa.24.413 Medline

35. E. Trinkaus, A. P. Buzhilova, The death and burial of Sunghir 1. Int. J. Osteoarchaeol. 22, 655-666 (2012). doi:10.1002/0a.1227

36. R. White, in Before Lascaux: The Complex Record of the Early Upper Paleolithic, H. Knecht, A. Pike-Tay, R. White, Eds. (CRC Press, 1993), pp. 277-299.

37. L. W. Cowgill, M. B. Mednikova, A. P. Buzhilova, E. Trinkaus, The Sunghir 3 Upper Paleolithic juvenile: Pathology versus persistence in the Paleolithic. Int. J. Osteoarchaeol. 25, 176-187 (2015). doi:10.1002/0a.2273 
38. D. Guatelli-Steinberg, A. P. Buzhilova, E. Trinkaus, Developmental stress and survival among the Mid Upper Paleolithic Sunghir children: Dental enamel hypoplasias of Sunghir 2 and 3. Int. J. Osteoarchaeol. 23, 421-431 (2013). doi:10.1002/oa.1263

39. B. Pinilla, E. Trinkaus, Buccal dental microwear and diet of the Sunghir Upper Paleolithic modern humans. Archaeol. Ethnol. Anthropol. Eurasia 42, 131-142 (2014). doi:10.1016/j.aeae.2015.01.013

40. A. P. Buzhilova, in Homo sungirensis. Upper Palaeolithic Man: Ecological and Evolutionary Aspects of the Investigation, T. I. Alexeeva, N. O. Bader, Eds. (Scientifc World, Moscow, 2000), pp. 302-314.

41. V. Formicola, A. P. Buzhilova, Double child burial from Sunghir (Russia): Pathology and inferences for upper paleolithic funerary practices. Am. J. Phys. Anthropol. 124, 189-198 (2004). doi:10.1002/ajpa.10273 Medline

42. V. Formicola, From the Sunghir children to the Romito dwarf. Curr. Anthropol. 48, 446-453 (2007). doi:10.1086/517592

43. J. Riel-Salvatore, C. Gravel-Miguel, in The Oxford Handbook of the Archaeology of Death and Burial (Oxford Univ. Press, 2013), pp. 303-346.

44. P. Pettitt, The Palaeolithic Origins of Human Burial (Routledge, 2011).

45. P. Mellars, Neanderthals and the modern human colonization of Europe. Nature 432, 461-465 (2004). doi:10.1038/nature03103 Medline

46. R. G. Klein, Out of Africa and the evolution of human behavior. Evol. Anthropol. 17, 267-281 (2008). doi:10.1002/evan.20181

47. J. A. Svoboda, O. Bar-Yosef, Stránská skála. Origins of the Upper Paleolithic in the Brno Basin, Moravia, Czech Republic (Peabody Museum of Archaeology and Ethnology, Harvard University, 2003).

48. G. B. Tostevin, Seeing Lithics: A Middle-Range Theory for Testing for Cultural Transmission in the Pleistocene (Oxbow, 2012).

49. P. R. Nigst, in Living in the Landscape: Essays in Honour of Graeme Barker, K. Boyle, R. J. Rabett, C. O. Hunt, Eds. (McDonald Institute for Archaeological Research, Cambridge, 2014), pp. 35-47.

50. P. R. Nigst, P. Haesaerts, F. Damblon, C. Frank-Fellner, C. Mallol, B. Viola, M. Götzinger, L. Niven, G. Trnka, J.-J. Hublin, Early modern human settlement of Europe north of the Alps occurred 43,500 years ago in a cold steppe-type environment. Proc. Natl. Acad. Sci. U.S.A. 111, 14394-14399 (2014). doi:10.1073/pnas.1412201111 Medline

51. M. D. Bosch, M. A. Mannino, A. L. Prendergast, T. C. O'Connell, B. Demarchi, S. M. Taylor, L. Niven, J. van der Plicht, J.-J. Hublin, New chronology for Ksâr 'Akil (Lebanon) supports Levantine route of modern human dispersal into Europe. Proc. NatI. Acad. Sci. U.S.A. 112, 7683-7688 (2015). doi:10.1073/pnas.1501529112 Medline

52. J. Zilhão, Neandertals and moderns mixed, and it matters. Evol. Anthropol. 15, 183-195 (2006). doi:10.1002/evan.20110

53. N. Teyssandier, Revolution or evolution: The emergence of the Upper Paleolithic in Europe. World Archaeol. 40, 493-519 (2008). doi:10.1080/00438240802452676

54. A. A. Sinitsyn, in The Chronology of the Aurignacian and of the Transitional Technocomplexes: Dating, Stratigraphies, Cultural Implications, J. Zilhao, F. d'Errico, Eds. (Instituto Português de Arqueologia, Lisboa, 2003), pp. 89-107.

55. E. Trinkaus, O. Moldovan, S. Milota, A. Bîlgăr, L. Sarcina, S. Athreya, S. E. Bailey, R. Rodrigo, G. Mircea, T. Higham, C. B. Ramsey, J. van der Plicht, An early modern human from the Peştera cu Oase, Romania. Proc. Natl. Acad. Sci. U.S.A. 100, 11231-11236 (2003). doi:10.1073/pnas.2035108100 Medline

56. J. Svoboda, The depositional context of the early upper paleolithic human fossils from the Koneprusy (Zlatý kůn) and Mladec caves, Czech republic. J. Hum. Evol.

\section{523-536 (2000). doi:10.1006/ihev.1999.0361 Medline}

57. M. White, P. Pettitt, Ancient digs and modern myths: The age and context of the Kent's Cavern 4 maxilla and the earliest Homo sapiens specimens in Europe. Eur. J. Archaeol. 15, 392-420 (2012). doi:10.1179/1461957112Y.0000000019

58. T. Higham, T. Compton, C. Stringer, R. Jacobi, B. Shapiro, E. Trinkaus, B. Chandler, F. Gröning, C. Collins, S. Hillson, P. O'Higgins, C. FitzGerald, M. Fagan, The earliest evidence for anatomically modern humans in northwestern Europe. Nature 479, 521-524 (2011). doi:10.1038/nature10484 Medline

59. C. Proctor, K. Douka, J. W. Proctor, T. Higham, The age and context of the KC4 maxilla, Kent's Cavern, UK. Eur. J. Archaeol. 20, 74-97 (2017) doi:10.1017/eaa.2016.1

60. S. Benazzi, K. Douka, C. Fornai, C. C. Bauer, O. Kullmer, J. Svoboda, I. Pap, F. Mallegni, P. Bayle, M. Coquerelle, S. Condemi, A. Ronchitelli, K. Harvati, G. W. Weber, Early dispersal of modern humans in Europe and implications for Neanderthal behaviour. Nature 479, 525-528 (2011). doi:10.1038/nature10617 Medline

61. W. E. Banks, F. d'Errico, J. Zilhão, Human-climate interaction during the Early Upper Paleolithic: Testing the hypothesis of an adaptive shift between the ProtoAurignacian and the Early Aurignacian. J. Hum. Evol. 64, 39-55 (2013). doi:10.1016/i.jhevol.2012.10.001 Medline

62. A. Ronchitelli, S. Benazzi, P. Boscato, K. Douka, A. Moroni, Comments on "Human-climate interaction during the Early Upper Paleolithic: Testing the hypothesis of an adaptive shift between the Proto-Aurignacian and the Early Aurignacian" by William E. Banks, Francesco d'Errico, João Zilhão. J. Hum. Evol. 73, 107-111 (2014). doi:10.1016/j.jhevol.2013.12.010 Medline

63. J. Zilhão, W. E. Banks, F. d'Errico, P. Gioia, Analysis of site formation and assemblage integrity does not support attribution of the Uluzzian to modern humans at Grotta del Cavallo. PLOS ONE 10, e0131181 (2015). doi:10.1371/journal.pone.0131181 Medline

64. J. A. Svoboda, V. Loek, E. Vlèek, Hunters Between East and West. The Paleolithic of Moravia (Plenum, 1996)

65. E. Trinkaus, J. Svoboda, Early Modern Human Evolution in Central Europe: The People of Dolní Vstonice and Pavlov (Oxford Univ. Press, 2006)

66. A. A. Sinitsyn, in La spiritualité (Etudes et Recherches Archeologiques de I'Universite de Liege), M. Otte, Ed. (2004), pp. 237-244.

67. V. Formicola, in Hominidae: Proceedings of the 2 nd International Congress of Human Paleontology (1989), pp. 483-486.

68. M. Mussi, On the chronology of the burials found in the Grimaldi Caves. Antropologia Contemporanea 9, 95-104 (1986).

69. P. B. Pettitt, M. Richards, R. Maggi, V. Formicola, The Gravettian burial known as the Prince ("Il Principe"): New evidence for his age and diet. Antiquity 77, 15-19 (2003). doi:10.1017/S0003598X00061305

70. J. Zilhão, E. Trinkaus, Portrait of the Artist as a Child. The Gravettian Human Skeleton from the Abrigo do Lagar Velho and its Archaeological Context (Instituto Português de Arqueologia, Lisboa, 2002).

71. B. Klima, Dolní Vestonice II: Ein Mammutjägerplatz und seine Bestattungen (Etudes et Recherches Archeologiques de l'Universite de Liege) (1995).

72. T. Einwögerer, H. Friesinger, M. Händel, C. Neugebauer-Maresch, U. Simon, M. Teschler-Nicola, Upper Palaeolithic infant burials. Nature 444, 285 (2006). doi:10.1038/444285a Medline

73. U. Simon, M. Händel, T. Einwögerer, C. Neugebauer-Maresch, The archaeological record of the Gravettian open air site Krems-Wachtberg. Quat. Int. 351, 5-13 (2014). doi:10.1016/j.quaint.2013.08.009

74. J. A Svoboda, The upper paleolithic burial area at Predmostí: Ritual and 
taphonomy. J. Hum. Evol. 54, 15-33 (2008). doi:10.1016/i.jhevol.2007.05.016 Medline

75. W. Antl-Weiser, M. Teschler-Nicola, Die menschlichen Zahnfunde von der Gravvettienfundstelle Grub/Kranawetberg bei Stillfried an der March, Niederösterreich. Archaeol. Austriaca 84-85, 201-212 (2000).

76. M. E. Teschler-Nicola, W. Antl-Weiser, H. Prossinger, Two Gravettian human deciduous teeth from Grub/Kranawetberg, lower Austria. Homo 54, 229-239 (2004). doi:10.1078/0018-442X-00074 Medline

77. C. Vercoutère, G. Giacobini, M. Patou-Mathis, Une dent humaine perforée découverte en contexte Gravettien ancien à l'abri Pataud (Dordogne, France). Anthropologie 112, 273-283 (2008). doi:10.1016/j.anthro.2008.02.002

78. P. B. Damgaard, A. Margaryan, H. Schroeder, L. Orlando, E. Willerslev, M. E. Allentoft, Improving access to endogenous DNA in ancient bones and teeth. Sci. Rep. 5, 11184 (2015). doi:10.1038/srep11184 Medline

79. J. Dabney, M. Knapp, I. Glocke, M.-T. Gansauge, A. Weihmann, B. Nickel, C. Valdiosera, N. García, S. Pääbo, J.-L. Arsuaga, M. Meyer, Complete mitochondrial genome sequence of a Middle Pleistocene cave bear reconstructed from ultrashort DNA fragments. Proc. Natl. Acad. Sci. U.S.A. 110, 15758-15763 (2013). doi:10.1073/pnas.1314445110 Medline

80. D. Y. Yang, B. Eng, J. S. Waye, J. C. Dudar, S. R. Saunders, Improved DNA extraction from ancient bones using silica-based spin columns. Am. J. Phys. Anthropol. 105, 539-543 (1998). doi:10.1002/(SICI)10968644(199804)105:4<539:AID-AJPA10>3.0.C0:2-1 Medline

81. C. Gamba, E. R. Jones, M. D. Teasdale, R. L. McLaughlin, G. Gonzalez-Fortes, V. Mattiangeli, L. Domboróczki, I. Kővári, I. Pap, A. Anders, A. Whittle, J. Dani, P. Raczky, T. F. G. Higham, M. Hofreiter, D. G. Bradley, R. Pinhasi, Genome flux and stasis in a five millennium transect of European prehistory. Nat. Commun. 5 , 5257 (2014). doi:10.1038/ncomms6257 Medline

82. M. E. Allentoft, M. Sikora, K.-G. Sjögren, S. Rasmussen, M. Rasmussen, J. Stenderup, P. B. Damgaard, H. Schroeder, T. Ahlström, L. Vinner, A.-S. Malaspinas, A. Margaryan, T. Higham, D. Chivall, N. Lynnerup, L. Harvig, J. Baron, P. Della Casa, P. Dąbrowski, P. R. Duffy, A. V. Ebel, A. Epimakhov, K. Frei, M. Furmanek, T. Gralak, A. Gromov, S. Gronkiewicz, G. Grupe, T. Hajdu, R. Jarysz, V. Khartanovich, A. Khokhlov, V. Kiss, J. Kolář, A. Kriiska, I. Lasak, C. Longhi, G. McGlynn, A. Merkevicius, I. Merkyte, M. Metspalu, R. Mkrtchyan, V. Moiseyev, L. Paja, G. Pálfi, D. Pokutta, Ł. Pospieszny, T. D. Price, L. Saag, M. Sablin, N. Shishlina, V. Smrčka, V. I. Soenov, V. Szeverényi, G. Tóth, S. V. Trifanova, L. Varul, M. Vicze, L. Yepiskoposyan, V. Zhitenev, L. Orlando, T. Sicheritz-Pontén, S. Brunak, R. Nielsen, K. Kristiansen, E. Willerslev, Population genomics of Bronze Age Eurasia. Nature 522, 167-172 (2015). doi:10.1038/nature14507 Medline

83. M. Meyer, M. Kircher, Illumina sequencing library preparation for highly multiplexed target capture and sequencing. Cold Spring Harb. Protoc. (2010). doi:10.1101/pdb.prot5448

84. A. Seguin-Orlando, M. Schubert, J. Clary, J. Stagegaard, M. T. Alberdi, J. L. Prado, A. Prieto, E. Willerslev, L. Orlando, Ligation bias in Illumina next-generation DNA libraries: Implications for sequencing ancient genomes. PLOS ONE 8, e78575 (2013). doi:10.1371/journal.pone.0078575 Medline

85. A. W. Briggs, U. Stenzel, P. L. F. Johnson, R. E. Green, J. Kelso, K. Prüfer, M. Meyer, J. Krause, M. T. Ronan, M. Lachmann, S. Pääbo, Patterns of damage in genomic DNA sequences from a Neandertal. Proc. NatI. Acad. Sci. U.S.A. 104, 14616-14621 (2007). doi:10.1073/pnas.0704665104 Medline

86. A. W. Briggs, U. Stenzel, M. Meyer, J. Krause, M. Kircher, S. Pääbo, Removal of deaminated cytosines and detection of in vivo methylation in ancient DNA. Nucleic Acids Res. 38, e87 (2010). doi:10.1093/nar/gkp1163 Medline

87. M. Schubert, L. Ermini, C. Der Sarkissian, H. Jónsson, A. Ginolhac, R. Schaefer, M. D. Martin, R. Fernández, M. Kircher, M. McCue, E. Willerslev, L. Orlando, Characterization of ancient and modern genomes by SNP detection and phylogenomic and metagenomic analysis using PALEOMIX. Nat. Protoc. 9 , 1056-1082 (2014). doi:10.1038/nprot.2014.063 Medline

88. J. T. Vilstrup, A. Seguin-Orlando, M. Stiller, A. Ginolhac, M. Raghavan, S. C. A. Nielsen, J. Weinstock, D. Froese, S. K. Vasiliev, N. D. Ovodov, J. Clary, K. M. Helgen, R. C. Fleischer, A. Cooper, B. Shapiro, L. Orlando, Mitochondrial phylogenomics of modern and ancient equids. PLOS ONE 8, e55950 (2013). doi:10.1371/journal.pone.0055950 Medline

89. M. Schubert, A. Ginolhac, S. Lindgreen, J. F. Thompson, K. A. S. Al-Rasheid, E. Willerslev, A. Krogh, L. Orlando, Improving ancient DNA read mapping against modern reference genomes. BMC Genomics 13, 178 (2012). doi:10.1186/14712164-13-178 Medline

90. S. Lindgreen, AdapterRemoval: Easy cleaning of next-generation sequencing reads. BMC Res. Notes 5, 337 (2012). doi:10.1186/1756-0500-5-337 Medline

91. H. Li, R. Durbin, Fast and accurate short read alignment with Burrows-Wheeler transform. Bioinformatics 25, 1754-1760 (2009). doi:10.1093/bioinformatics/btp324 Medline

92. A. McKenna, M. Hanna, E. Banks, A. Sivachenko, K. Cibulskis, A. Kernytsky, K. Garimella, D. Altshuler, S. Gabriel, M. Daly, M. A. DePristo, The Genome Analysis Toolkit: A MapReduce framework for analyzing next-generation DNA sequencing data. Genome Res. 20, 1297-1303 (2010). doi:10.1101/gr.107524.110 Medline

93. H. Jónsson, A. Ginolhac, M. Schubert, P. L. F. Johnson, L. Orlando, mapDamage2.0: Fast approximate Bayesian estimates of ancient DNA damage parameters. Bioinformatics 29, 1682-1684 (2013). doi:10.1093/bioinformatics/btt193 Medline

94. P. Skoglund, J. Storå, A. Götherström, M. Jakobsson, Accurate sex identification of ancient human remains using DNA shotgun sequencing. J. Archaeol. Sci. 40, 4477-4482 (2013). doi:10.1016/j.jas.2013.07.004

95. T. S. Korneliussen, A. Albrechtsen, R. Nielsen, ANGSD: Analysis of Next Generation Sequencing Data. BMC Bioinformatics 15, 356 (2014). doi:10.1186/s12859-014-0356-4 Medline

96. M. Rasmussen, X. Guo, Y. Wang, K. E. Lohmueller, S. Rasmussen, A. Albrechtsen, L. Skotte, S. Lindgreen, M. Metspalu, T. Jombart, T. Kivisild, W. Zhai, A. Eriksson, A. Manica, L. Orlando, F. M. De La Vega, S. Tridico, E. Metspalu, K. Nielsen, M. C. Ávila-Arcos, J. V. Moreno-Mayar, C. Muller, J. Dortch, M. T. P. Gilbert, O. Lund, A. Wesolowska, M. Karmin, L. A. Weinert, B. Wang, J. Li, S. Tai, F. Xiao, T. Hanihara, G. van Driem, A. R. Jha, F.-X. Ricaut, P. de Knijff, A. B. Migliano, I. Gallego Romero, K. Kristiansen, D. M. Lambert, S. Brunak, P. Forster, B. Brinkmann, 0. Nehlich, M. Bunce, M. Richards, R. Gupta, C. D. Bustamante, A. Krogh, R. A. Foley, M. M. Lahr, F. Balloux, T. Sicheritz-Pontén, R. Villems, R. Nielsen, J. Wang, E. Willerslev, An Aboriginal Australian genome reveals separate human dispersals into Asia. Science 334, 94-98 (2011). doi:10.1126/science.1211177 Medline

97. 1000 Genomes Project Consortium, An integrated map of genetic variation from 1,092 human genomes. Nature 491, 56-65 (2012). doi:10.1038/nature11632 Medline

98. M. Stuiver, H. A. Polach, Discussion reporting of ${ }^{14} \mathrm{C}$ data. Radiocarbon $19,355-$ 363 (1977). doi:10.1017/S0033822200003672

99. F. Brock, T. Higham, P. Ditchfield, C. B. Ramsey, Current pretreatment methods for AMS radiocarbon dating at the Oxford Radiocarbon Accelerator Unit (ORAU). Radiocarbon 52, 103-112 (2010). doi:10.1017/S0033822200045069

100. P. J. Reimer, M. G. L. Baillie, E. Bard, A. Bayliss, J. W. Beck, P. G. Blackwell, C. Bronk Ramsey, C. E. Buck, G. S. Burr, R. L. Edwards, M. Friedrich, P. M. Grootes, T. P. Guilderson, I. Hajdas, T. J. Heaton, A. G. Hogg, K. A. Hughen, K. F. Kaiser, B. Kromer, F. G. McCormac, S. W. Manning, R. W. Reimer, D. A. Richards, J. R. Southon, S. Talamo, C. S. M. Turney, J. van der Plicht, C. E. Weyhenmeyer, IntCal09 and Marine09 radiocarbon age calibration curves, 0-50,000 years cal BP. Radiocarbon 51, 1111-1150 (2009). doi:10.1017/S0033822200034202 
101. S. Nalawade-Chavan, J. McCullagh, R. Hedges, C. Bonsall, A. Boroneanţ, C. B. Ramsey, T. Higham, Compound-specific radiocarbon dating of essential and non-essential amino acids: Towards determination of dietary reservoir effects in humans. Radiocarbon 55, 709-719 (2013). doi:10.1017/S0033822200057866

102. H. Li, B. Handsaker, A. Wysoker, T. Fennell, J. Ruan, N. Homer, G. Marth, G. Abecasis, R. Durbin, The Sequence Alignment/Map format and SAMtools. Bioinformatics 25, 2078-2079 (2009). doi:10.1093/bioinformatics/btp352 Medline

103. H. Li, A statistical framework for SNP calling, mutation discovery, association mapping and population genetical parameter estimation from sequencing data. Bioinformatics 27, 2987-2993 (2011). doi:10.1093/bioinformatics/btr509 Medline

104. W. Haak, I. Lazaridis, N. Patterson, N. Rohland, S. Mallick, B. Llamas, G. Brandt, S. Nordenfelt, E. Harney, K. Stewardson, Q. Fu, A. Mittnik, E. Bánffy, C. Economou, M. Francken, S. Friederich, R. G. Pena, F. Hallgren, V. Khartanovich, A. Khokhlov, M. Kunst, P. Kuznetsov, H. Meller, O. Mochalov, V. Moiseyev, N. Nicklisch, S. L. Pichler, R. Risch, M. A. Rojo Guerra, C. Roth, A. Szécsényi-Nagy, J. Wahl, M. Meyer, J. Krause, D. Brown, D. Anthony, A. Cooper, K. W. Alt, D. Reich, Massive migration from the steppe was a source for Indo-European languages in Europe. Nature 522, 207-211 (2015). doi:10.1038/nature14317 Medline

105. Q. Fu, M. Hajdinjak, O. T. Moldovan, S. Constantin, S. Mallick, P. Skoglund, N. Patterson, N. Rohland, I. Lazaridis, B. Nickel, B. Viola, K. Prüfer, M. Meyer, J. Kelso, D. Reich, S. Pääbo, An early modern human from Romania with a recent Neanderthal ancestor. Nature 524, 216-219 (2015). doi:10.1038/nature14558 Medline

106. M. Raghavan, P. Skoglund, K. E. Graf, M. Metspalu, A. Albrechtsen, I. Moltke, S. Rasmussen, T. W. Stafford Jr., L. Orlando, E. Metspalu, M. Karmin, K. Tambets, S. Rootsi, R. Mägi, P. F. Campos, E. Balanovska, O. Balanovsky, E. Khusnutdinova, S. Litvinov, L. P. Osipova, S. A. Fedorova, M. I. Voevoda, M. DeGiorgio, T. Sicheritz-Ponten, S. Brunak, S. Demeshchenko, T. Kivisild, R. Villems, R. Nielsen, M. Jakobsson, E. Willerslev, Upper Palaeolithic Siberian genome reveals dual ancestry of Native Americans. Nature 505, 87-91 (2014). doi:10.1038/nature12736 Medline

107. P. Skoglund, H. Malmström, A. Omrak, M. Raghavan, C. Valdiosera, T. Günther, P. Hall, K. Tambets, J. Parik, K.-G. Sjögren, J. Apel, E. Willerslev, J. Storå, A. Götherström, M. Jakobsson, Genomic diversity and admixture differs for StoneAge Scandinavian foragers and farmers. Science 344, 747-750 (2014). doi:10.1126/science.1253448 Medline

108. E. R. Jones, G. Gonzalez-Fortes, S. Connell, V. Siska, A. Eriksson, R. Martiniano, R. L. McLaughlin, M. Gallego Llorente, L. M. Cassidy, C. Gamba, T. Meshveliani, O. Bar-Yosef, W. Müller, A. Belfer-Cohen, Z. Matskevich, N. Jakeli, T. F. G. Higham, M. Currat, D. Lordkipanidze, M. Hofreiter, A. Manica, R. Pinhasi, D. G. Bradley, Upper Palaeolithic genomes reveal deep roots of modern Eurasians. Nat. Commun. 6, 8912 (2015). doi:10.1038/ncomms9912 Medline

109. I. Mathieson, I. Lazaridis, N. Rohland, S. Mallick, N. Patterson, S. A. Roodenberg, E. Harney, K. Stewardson, D. Fernandes, M. Novak, K. Sirak, C. Gamba, E. R. Jones, B. Llamas, S. Dryomov, J. Pickrell, J. L. Arsuaga, J. M. B. de Castro, E. Carbonell, F. Gerritsen, A. Khokhlov, P. Kuznetsov, M. Lozano, H. Meller, O. Mochalov, V. Moiseyev, M. A. R. Guerra, J. Roodenberg, J. M. Vergès, J. Krause, A. Cooper, K. W. Alt, D. Brown, D. Anthony, C. Lalueza-Fox, W. Haak, R. Pinhasi, D. Reich, Genome-wide patterns of selection in 230 ancient Eurasians. Nature 528, 499-503 (2015). doi:10.1038/nature16152 Medline

110. I. Olalde, H. Schroeder, M. Sandoval-Velasco, L. Vinner, I. Lobón, O. Ramirez, S Civit, P. García Borja, D. C. Salazar-García, S. Talamo, J. María Fullola, F. Xavier Oms, M. Pedro, P. Martínez, M. Sanz, J. Daura, J. Zilhão, T. Marquès-Bonet, M. T. Gilbert, C. Lalueza-Fox, A common genetic origin for early farmers from Mediterranean Cardial and Central European LBK cultures. Mol. Biol. Evol. 32 , 3132-3142 (2015). doi:10.1093/molbev/msv181 Medline

111. I. Lazaridis, D. Nadel, G. Rollefson, D. C. Merrett, N. Rohland, S. Mallick, D.
Fernandes, M. Novak, B. Gamarra, K. Sirak, S. Connell, K. Stewardson, E. Harney, Q. Fu, G. Gonzalez-Fortes, E. R. Jones, S. A. Roodenberg, G. Lengyel, F. Bocquentin, B. Gasparian, J. M. Monge, M. Gregg, V. Eshed, A.-S. Mizrahi, C. Meiklejohn, F. Gerritsen, L. Bejenaru, M. Blüher, A. Campbell, G. Cavalleri, D. Comas, P. Froguel, E. Gilbert, S. M. Kerr, P. Kovacs, J. Krause, D. McGettigan, M. Merrigan, D. A. Merriwether, S. O'Reilly, M. B. Richards, O. Semino, M. ShamoonPour, G. Stefanescu, M. Stumvoll, A. Tönjes, A. Torroni, J. F. Wilson, L. Yengo, N. A. Hovhannisyan, N. Patterson, R. Pinhasi, D. Reich, Genomic insights into the origin of farming in the ancient Near East. Nature 536, 419-424 (2016). doi:10.1038/nature19310 Medline

112. F. Broushaki, M. G. Thomas, V. Link, S. López, L. van Dorp, K. Kirsanow, Z. Hofmanová, Y. Diekmann, L. M. Cassidy, D. Díez-Del-Molino, A. Kousathanas, C. Sell, H. K. Robson, R. Martiniano, J. Blöcher, A. Scheu, S. Kreutzer, R. Bollongino, D. Bobo, H. Davudi, O. Munoz, M. Currat, K. Abdi, F. Biglari, O. E. Craig, D. G. Bradley, S. Shennan, K. Veeramah, M. Mashkour, D. Wegmann, G. Hellenthal, J. Burger, Early Neolithic genomes from the eastern Fertile Crescent. Science 353 , 499-503 (2016). doi:10.1126/science.aaf7943 Medline

113. Z. Hofmanová, S. Kreutzer, G. Hellenthal, C. Sell, Y. Diekmann, D. Díez-DelMolino, L. van Dorp, S. López, A. Kousathanas, V. Link, K. Kirsanow, L. M. Cassidy, R. Martiniano, M. Strobel, A. Scheu, K. Kotsakis, P. Halstead, S. Triantaphyllou, N. Kyparissi-Apostolika, D. Urem-Kotsou, C. Ziota, F. Adaktylou, S. Gopalan, D. M. Bobo, L. Winkelbach, J. Blöcher, M. Unterländer, C. Leuenberger, C.. Çilingiroğlu, B. Horejs, F. Gerritsen, S. J. Shennan, D. G. Bradley, M. Currat, K. R. Veeramah, D. Wegmann, M. G. Thomas, C. Papageorgopoulou, J. Burger, Early farmers from across Europe directly descended from Neolithic Aegeans. Proc. Natl. Acad. Sci. U.S.A. 113, 6886-6891 (2016). doi:10.1073/pnas.1523951113 Medline

114. G. M. Kılınç, A. Omrak, F. Özer, T. Günther, A. M. Büyükkarakaya, E. Bıçakçı, D. Baird, H. M. Dönertaş, A. Ghalichi, R. Yaka, D. Koptekin, S. C. Açan, P. Parvizi, M. Krzewińska, E. A. Daskalaki, E. Yüncü, N. D. Dağtaş, A. Fairbairn, J. Pearson, G. Mustafaoğlu, Y. S. Erdal, Y. G. Çakan, I. Togan, M. Somel, J. Storå, M. Jakobsson, A. Götherström, The demographic development of the first farmers in Anatolia. Curr. Biol. 26, 2659-2666 (2016). doi:10.1016/i.cub.2016.07.057 Medline

115. S. Mallick, H. Li, M. Lipson, I. Mathieson, M. Gymrek, F. Racimo, M. Zhao, N. Chennagiri, S. Nordenfelt, A. Tandon, P. Skoglund, I. Lazaridis, S. Sankararaman, Q. Fu, N. Rohland, G. Renaud, Y. Erlich, T. Willems, C. Gallo, J. P. Spence, Y. S. Song, G. Poletti, F. Balloux, G. van Driem, P. de Knijff, I. G. Romero, A. R. Jha, D. M. Behar, C. M. Bravi, C. Capelli, T. Hervig, A. Moreno-Estrada, O. L. Posukh, E. Balanovska, O. Balanovsky, S. Karachanak-Yankova, H. Sahakyan, D. Toncheva, L. Yepiskoposyan, C. Tyler-Smith, Y. Xue, M. S. Abdullah, A. Ruiz-Linares, C. M. Beall, A. Di Rienzo, C. Jeong, E. B. Starikovskaya, E. Metspalu, J. Parik, R. Villems, B. M. Henn, U. Hodoglugil, R. Mahley, A. Sajantila, G. Stamatoyannopoulos, J. T. S. Wee, R. Khusainova, E. Khusnutdinova, S. Litvinov, G. Ayodo, D. Comas, M. F. Hammer, T. Kivisild, W. Klitz, C. A. Winkler, D. Labuda, M. Bamshad, L. B. Jorde, S. A. Tishkoff, W. S. Watkins, M. Metspalu, S. Dryomov, R. Sukernik, L. Singh, K. Thangaraj, S. Pääbo, J. Kelso, N. Patterson, D. Reich, The Simons Genome Diversity Project: 300 genomes from 142 diverse populations. Nature 538, 201206 (2016). doi:10.1038/nature18964 Medline

116. A.-S. Malaspinas, M. C. Westaway, C. Muller, V. C. Sousa, O. Lao, I. Alves, A. Bergström, G. Athanasiadis, J. Y. Cheng, J. E. Crawford, T. H. Heupink, E. Macholdt, S. Peischl, S. Rasmussen, S. Schiffels, S. Subramanian, J. L. Wright, A. Albrechtsen, C. Barbieri, I. Dupanloup, A. Eriksson, A. Margaryan, I. Moltke, I. Pugach, T. S. Korneliussen, I. P. Levkivskyi, J. V. Moreno-Mayar, S. Ni, F. Racimo, M. Sikora, Y. Xue, F. A. Aghakhanian, N. Brucato, S. Brunak, P. F. Campos, W. Clark, S. Ellingvåg, G. Fourmile, P. Gerbault, D. Injie, G. Koki, M. Leavesley, B. Logan, A. Lynch, E. A. Matisoo-Smith, P. J. McAllister, A. J. Mentzer, M. Metspalu, A. B. Migliano, L. Murgha, M. E. Phipps, W. Pomat, D. Reynolds, F.-X. Ricaut, P. Siba, M. G. Thomas, T. Wales, C. M. Wall, S. J. Oppenheimer, C. Tyler-Smith, R. Durbin, J. Dortch, A. Manica, M. H. Schierup, R. A. Foley, M. M. Lahr, C. Bowern, J. D. Wall, T. Mailund, M. Stoneking, R. Nielsen, M. S. Sandhu, L. Excoffier, D. M. Lambert, E. Willerslev, A genomic history of Aboriginal Australia. Nature 538, 207-214 (2016). doi:10.1038/nature18299 Medline 
117. M. Mondal, F. Casals, T. Xu, G. M. Dall'Olio, M. Pybus, M. G. Netea, D. Comas, H. Laayouni, Q. Li, P. P. Majumder, J. Bertranpetit, Genomic analysis of Andamanese provides insights into ancient human migration into Asia and adaptation. Nat. Genet. 48, 1066-1070 (2016). doi:10.1038/ng.3621 Medline

118. M. Raghavan, M. DeGiorgio, A. Albrechtsen, I. Moltke, P. Skoglund, T. S. Korneliussen, B. Grønnow, M. Appelt, H. C. Gulløv, T. M. Friesen, W. Fitzhugh, H. Malmström, S. Rasmussen, J. Olsen, L. Melchior, B. T. Fuller, S. M. Fahrni, T. Stafford Jr., V. Grimes, M. A. P. Renouf, J. Cybulski, N. Lynnerup, M. M. Lahr, K. Britton, R. Knecht, J. Arneborg, M. Metspalu, O. E. Cornejo, A.-S. Malaspinas, Y. Wang, M. Rasmussen, V. Raghavan, T. V. O. Hansen, E. Khusnutdinova, T. Pierre, K. Dneprovsky, C. Andreasen, H. Lange, M. G. Hayes, J. Coltrain, V. A. Spitsyn, A. Götherström, L. Orlando, T. Kivisild, R. Villems, M. H. Crawford, F. C. Nielsen, J. Dissing, J. Heinemeier, M. Meldgaard, C. Bustamante, D. H. O'Rourke, M. Jakobsson, M. T. P. Gilbert, R. Nielsen, E. Willerslev, The genetic prehistory of the New World Arctic. Science 345, 1255832 (2014). doi:10.1126/science.1255832 Medline

119. M. Raghavan, M. Steinrücken, K. Harris, S. Schiffels, S. Rasmussen, M. DeGiorgio, A. Albrechtsen, C. Valdiosera, M. C. Ávila-Arcos, A.-S. Malaspinas, A. Eriksson, I. Moltke, M. Metspalu, J. R. Homburger, J. Wall, O. E. Cornejo, J. V. Moreno-Mayar, T. S. Korneliussen, T. Pierre, M. Rasmussen, P. F. Campos, P. de Barros Damgaard, M. E. Allentoft, J. Lindo, E. Metspalu, R. Rodríguez-Varela, J. Mansilla, C. Henrickson, A. Seguin-Orlando, H. Malmström, T. Stafford Jr., S. S. Shringarpure, A. Moreno-Estrada, M. Karmin, K. Tambets, A. Bergström, Y. Xue, V. Warmuth, A. D. Friend, J. Singarayer, P. Valdes, F. Balloux, I. Leboreiro, J. L. Vera, H. Rangel-Villalobos, D. Pettener, D. Luiselli, L. G. Davis, E. Heyer, C. P. E. Zollikofer, M. S. Ponce de León, C. I. Smith, V. Grimes, K.-A. Pike, M. Deal, B. T. Fuller, B. Arriaza, V. Standen, M. F. Luz, F. Ricaut, N. Guidon, L. Osipova, M. I. Voevoda, O. L. Posukh, O. Balanovsky, M. Lavryashina, Y. Bogunov, E. Khusnutdinova, M. Gubina, E. Balanovska, S. Fedorova, S. Litvinov, B. Malyarchuk, M. Derenko, M. J. Mosher, D. Archer, J. Cybulski, B. Petzelt, J. Mitchell, R. Worl, P. J. Norman, P. Parham, B. M. Kemp, T. Kivisild, C. TylerSmith, M. S. Sandhu, M. Crawford, R. Villems, D. G. Smith, M. R. Waters, T. Goebel, J. R. Johnson, R. S. Malhi, M. Jakobsson, D. J. Meltzer, A. Manica, R. Durbin, C. D. Bustamante, Y. S. Song, R. Nielsen, E. Willerslev, Genomic evidence for the Pleistocene and recent population history of Native Americans. Science 349, aab3884 (2015). doi:10.1126/science.aab3884 Medline

120. I. Lazaridis, N. Patterson, A. Mittnik, G. Renaud, S. Mallick, K. Kirsanow, P. H. Sudmant, J. G. Schraiber, S. Castellano, M. Lipson, B. Berger, C. Economou, R. Bollongino, Q. Fu, K. I. Bos, S. Nordenfelt, H. Li, C. de Filippo, K. Prüfer, S. Sawyer, C. Posth, W. Haak, F. Hallgren, E. Fornander, N. Rohland, D. Delsate, M. Francken, J.-M. Guinet, J. Wahl, G. Ayodo, H. A. Babiker, G. Bailliet, E. Balanovska, O. Balanovsky, R. Barrantes, G. Bedoya, H. Ben-Ami, J. Bene, F. Berrada, C. M. Bravi, F. Brisighelli, G. B. J. Busby, F. Cali, M. Churnosov, D. E. C. Cole, D. Corach, L. Damba, G. van Driem, S. Dryomov, J.-M. Dugoujon, S. A. Fedorova, I. Gallego Romero, M. Gubina, M. Hammer, B. M. Henn, T. Hervig, U. Hodoglugil, A. R. Jha, S. Karachanak-Yankova, R. Khusainova, E. Khusnutdinova, R. Kittles, T. Kivisild, W. Klitz, V. Kučinskas, A. Kushniarevich, L. Laredj, S. Litvinov, T. Loukidis, R. W. Mahley, B. Melegh, E. Metspalu, J. Molina, J. Mountain, K. Näkkäläjärvi, D. Nesheva, T. Nyambo, L. Osipova, J. Parik, F. Platonov, O. Posukh, V. Romano, F. Rothhammer, I. Rudan, R. Ruizbakiev, H. Sahakyan, A. Sajantila, A. Salas, E. B. Starikovskaya, A. Tarekegn, D. Toncheva, S. Turdikulova, I. Uktveryte, O. Utevska, R. Vasquez, M. Villena, M. Voevoda, C. A. Winkler, L. Yepiskoposyan, P. Zalloua, T. Zemunik, A. Cooper, C. Capelli, M. G. Thomas, A. Ruiz-Linares, S. A. Tishkoff, L. Singh, K. Thangaraj, R. Villems, D. Comas, R. Sukernik, M. Metspalu, M. Meyer, E. E. Eichler, J. Burger, M. Slatkin, S. Pääbo, J. Kelso, D. Reich, J. Krause, Ancient human genomes suggest three ancestral populations for present-day Europeans. Nature 513, 409-413 (2014). doi:10.1038/nature13673 Medline

121. R. E. Green, J. Krause, A. W. Briggs, T. Maricic, U. Stenzel, M. Kircher, N. Patterson, H. Li, W. Zhai, M. H. Y. Fritz, N. F. Hansen, E. Y. Durand, A. S. Malaspinas, J. D. Jensen, T. Marques-Bonet, C. Alkan, K. Prüfer, M. Meyer, H. A. Burbano, J. M. Good, R. Schultz, A. Aximu-Petri, A. Butthof, B. Höber, B. Höffner, M. Siegemund, A. Weihmann, C. Nusbaum, E. S. Lander, C. Russ, N. Novod, J.
Affourtit, M. Egholm, C. Verna, P. Rudan, D. Brajkovic, Ž. Kucan, I. Gušic, V. B. Doronichev, L. V. Golovanova, C. Lalueza-Fox, M. de la Rasilla, J. Fortea, A. Rosas, R. W. Schmitz, P. L. F. Johnson, E. E. Eichler, D. Falush, E. Birney, J. C. Mullikin, M. Slatkin, R. Nielsen, J. Kelso, M. Lachmann, D. Reich, S. Pääbo, A draft sequence of the Neandertal genome. Science 328, 710-722 (2010). doi:10.1126/science. 1188021 Medline

122. G. Renaud, V. Slon, A. T. Duggan, J. Kelso, Schmutzi: Estimation of contamination and endogenous mitochondrial consensus calling for ancient DNA. Genome Biol. 16, 224 (2015). doi:10.1186/s13059-015-0776-0 Medline

123. H. Weissensteiner, D. Pacher, A. Kloss-Brandstätter, L. Forer, G. Specht, H.-J. Bandelt, F. Kronenberg, A. Salas, S. Schönherr, HaploGrep 2: Mitochondrial haplogroup classification in the era of high-throughput sequencing. Nucleic Acids Res. 44, W58-W63 (2016). doi:10.1093/nar/gkw233 Medline

124. A. Löytynoja, N. Goldman, Phylogeny-aware gap placement prevents errors in sequence alignment and evolutionary analysis. Science 320, 1632-1635 (2008). doi:10.1126/science. 1158395 Medline

125. A. Stamatakis, RAxML version 8: A tool for phylogenetic analysis and postanalysis of large phylogenies. Bioinformatics 30, 1312-1313 (2014). doi:10.1093/bioinformatics/btu033 Medline

126. I. Olalde, M. E. Allentoft, F. Sánchez-Quinto, G. Santpere, C. W. K. Chiang, M. DeGiorgio, J. Prado-Martinez, J. A. Rodríguez, S. Rasmussen, J. Quilez, O. Ramírez, U. M. Marigorta, M. Fernández-Callejo, M. E. Prada, J. M. V. Encinas, R. Nielsen, M. G. Netea, J. Novembre, R. A. Sturm, P. Sabeti, T. Marquès-Bonet, A. Navarro, E. Willerslev, C. Lalueza-Fox, Derived immune and ancestral pigmentation alleles in a 7,000-year-old Mesolithic European. Nature 507, 225228 (2014). doi:10.1038/nature12960 Medline

127. P. Hallast, C. Batini, D. Zadik, P. Maisano Delser, J. H. Wetton, E. Arroyo-Pardo, G. L. Cavalleri, P. de Knijff, G. Destro Bisol, B. M. Dupuy, H. A. Eriksen, L. B. Jorde, T. E. King, M. H. Larmuseau, A. López de Munain, A. M. López-Parra, A. Loutradis, J. Milasin, A. Novelletto, H. Pamjav, A. Sajantila, W. Schempp, M. Sears, A. Tolun, C. Tyler-Smith, A. Van Geystelen, S. Watkins, B. Winney, M. A. Jobling, The Ychromosome tree bursts into leaf: 13,000 high-confidence SNPs covering the majority of known clades. Mol. Biol. Evol. 32, 661-673 (2015). doi:10.1093/molbev/msu327 Medline

128. K. Tamura, G. Stecher, D. Peterson, A. Filipski, S. Kumar, MEGA6: Molecular Evolutionary Genetics Analysis version 6.0. Mol. Biol. Evol. 30, 2725-2729 (2013). doi:10.1093/molbev/mst197 Medline

129. T. S. Korneliussen, I. Moltke, NgsRelate: A software tool for estimating pairwise relatedness from next-generation sequencing data. Bioinformatics 31, 40094011 (2015). doi:10.1093/bioinformatics/btv509 Medline

130. A. Albrechtsen, T. Sand Korneliussen, I. Moltke, T. van Overseem Hansen, F. C. Nielsen, R. Nielsen, Relatedness mapping and tracts of relatedness for genomewide data in the presence of linkage disequilibrium. Genet. Epidemiol. 33, 266274 (2009). doi:10.1002/gepi.20378 Medline

131. A. Manichaikul, J. C. Mychaleckyj, S. S. Rich, K. Daly, M. Sale, W.-M. Chen, Robust relationship inference in genome-wide association studies. Bioinformatics 26, 2867-2873 (2010). doi:10.1093/bioinformatics/bta559 Medline

132. A. Seguin-Orlando, C. Gamba, C. Der Sarkissian, L. Ermini, G. Louvel, E. Boulygina, A. Sokolov, A. Nedoluzhko, E. D. Lorenzen, P. Lopez, H. G. McDonald, E. Scott, A. Tikhonov, T. W. Stafford Jr., A. H. Alfarhan, S. A. Alquraishi, K. A. S. Al-Rasheid, B. Shapiro, E. Willerslev, E. Prokhortchouk, L. Orlando, Pros and cons of methylation-based enrichment methods for ancient DNA. Sci. Rep. 5, 11826 (2015). doi:10.1038/srep11826 Medline

133. A. Ko, R. Nielsen, Composite likelihood method for inferring local pedigrees. PLOS Genet. 13, e1006963 (2017). doi:10.1371/journal.pgen.1006963

134. S. Purcell, B. Neale, K. Todd-Brown, L. Thomas, M. A. R. Ferreira, D. Bender, J. 
Maller, P. Sklar, P. I. W. de Bakker, M. J. Daly, P. C. Sham, PLINK: A tool set for whole-genome association and population-based linkage analyses. Am. J. Hum. Genet. 81, 559-575 (2007). doi:10.1086/519795 Medline

135. 1000 Genomes Project Consortium, A global reference for human genetic variation. Nature 526, 68-74 (2015). doi:10.1038/nature15393 Medline

136. J. M. M. Kuhn, M. Jakobsson, T. Günther, Estimating genetic kin relationships in prehistoric populations. bioRxiv:100297 (2017).

137. International HapMap 3 Consortium, Integrating common and rare genetic variation in diverse human populations. Nature 467, 52-58 (2010) doi:10.1038/nature09298 Medline

138. A. Scally, R. Durbin, Revising the human mutation rate: Implications for understanding human evolution. Nat. Rev. Genet. 13, 745-753 (2012) doi:10.1038/nrg3295 Medline

139. B. L. Browning, S. R. Browning, Detecting identity by descent and estimating genotype error rates in sequence data. Am. J. Hum. Genet. 93, 840-851 (2013) doi:10.1016/i.aihg.2013.09.014 Medline

140. P. F. Palamara, T. Lencz, A. Darvasi, I. Pe'er, Length distributions of identity by descent reveal fine-scale demographic history. Am. J. Hum. Genet. 91, 809-822 (2012). doi:10.1016/j.ajhg.2012.08.030 Medline

141. N. Patterson, P. Moorjani, Y. Luo, S. Mallick, N. Rohland, Y. Zhan, T. Genschoreck, T. Webster, D. Reich, Ancient admixture in human history. Genetics 192, 1065-1093 (2012). doi:10.1534/genetics.112.145037 Medline

142. N. Patterson, A. L. Price, D. Reich, Population structure and eigenanalysis. PLOS Genet. 2, e190 (2006). doi:10.1371/journal.pgen.0020190 Medline

143. R Project for Statistical Computing; www.r-project.org/.

144. M. Rasmussen, S. L. Anzick, M. R. Waters, P. Skoglund, M. DeGiorgio, T. W. Stafford Jr., S. Rasmussen, I. Moltke, A. Albrechtsen, S. M. Doyle, G. D. Poznik, V. Gudmundsdottir, R. Yadav, A.-S. Malaspinas, S. S. White 5th, M. E. Allentoft, O. E. Cornejo, K. Tambets, A. Eriksson, P. D. Heintzman, M. Karmin, T. S. Korneliussen, D. J. Meltzer, T. L. Pierre, J. Stenderup, L. Saag, V. M. Warmuth, M. C. Lopes, R. S. Malhi, S. Brunak, T. Sicheritz-Ponten, I. Barnes, M. Collins, L. Orlando, F. Balloux, A. Manica, R. Gupta, M. Metspalu, C. D. Bustamante, M. Jakobsson, R. Nielsen, E. Willerslev, The genome of a Late Pleistocene human from a Clovis burial site in western Montana. Nature 506, 225-229 (2014). doi:10.1038/nature13025 Medline

145. R. Nielsen, T. Korneliussen, A. Albrechtsen, Y. Li, J. Wang, SNP calling, genotype calling, and sample allele frequency estimation from new-generation sequencing data. PLOS ONE 7, e37558 (2012). doi:10.1371/journal.pone.0037558 Medline

146. J. C. Nash, R. Varadhan, Unifying optimization algorithms to aid software system users: optimx for R. J. Stat. Softw. 43 , www.jstatsoft.org/article/view/v043i09/ (2011)

147. A. M. Adams, R. R. Hudson, Maximum-likelihood estimation of demographic parameters using the frequency spectrum of unlinked single-nucleotide polymorphisms. Genetics 168, 1699-1712 (2004). doi:10.1534/genetics.104.030171 Medline

148. R. Nielsen, Estimation of population parameters and recombination rates from single nucleotide polymorphisms. Genetics 154, 931-942 (2000). Medline

149. L. Excoffier, I. Dupanloup, E. Huerta-Sánchez, V. C. Sousa, M. Foll, Robust demographic inference from genomic and SNP data. PLOS Genet. 9, e1003905 (2013). doi:10.1371/journal.pgen.1003905 Medline

150. X.-L. Meng, D. B. Rubin, Maximum likelihood estimation via the ECM algorithm: A general framework. Biometrika 80, 267-278 (1993). doi:10.1093/biomet/80.2.267

151. R. Brent, Algorithms for Minimization Without Derivatives (Prentice-Hall, 1973).
152. F. Cunningham, M. R. Amode, D. Barrell, K. Beal, K. Billis, S. Brent, D. CarvalhoSilva, P. Clapham, G. Coates, S. Fitzgerald, L. Gil, C. G. Girón, L. Gordon, T. Hourlier, S. E. Hunt, S. H. Janacek, N. Johnson, T. Juettemann, A. K. Kähäri, S. Keenan, F. J. Martin, T. Maurel, W. McLaren, D. N. Murphy, R. Nag, B. Overduin, A. Parker, M. Patricio, E. Perry, M. Pignatelli, H. S. Riat, D. Sheppard, K. Taylor, A. Thormann, A. Vullo, S. P. Wilder, A. Zadissa, B. L. Aken, E. Birney, J. Harrow, R. Kinsella, M. Muffato, M. Ruffier, S. M. J. Searle, G. Spudich, S. J. Trevanion, A. Yates, D. R. Zerbino, P. Flicek, Ensembl 2015. Nucleic Acids Res. 43, D662-D669 (2015). doi:10.1093/nar/gku1010 Medline

153. K. R. Rosenbloom, J. Armstrong, G. P. Barber, J. Casper, H. Clawson, M. Diekhans, T. R. Dreszer, P. A. Fujita, L. Guruvadoo, M. Haeussler, R. A. Harte, S. Heitner, G. Hickey, A. S. Hinrichs, R. Hubley, D. Karolchik, K. Learned, B. T. Lee, C. H. Li, K. H. Miga, N. Nguyen, B. Paten, B. J. Raney, A. F. A. Smit, M. L. Speir, A. S. Zweig, D. Haussler, R. M. Kuhn, W. J. Kent, The UCSC Genome Browser database: 2015 update. Nucleic Acids Res. 43, D670-D681 (2015). doi:10.1093/nar/gku1177 Medline

154. P. Flicek, M. R. Amode, D. Barrell, K. Beal, S. Brent, Y. Chen, P. Clapham, G. Coates, S. Fairley, S. Fitzgerald, L. Gordon, M. Hendrix, T. Hourlier, N. Johnson, A. Kähäri, D. Keefe, S. Keenan, R. Kinsella, F. Kokocinski, E. Kulesha, P. Larsson, I. Longden, W. McLaren, B. Overduin, B. Pritchard, H. S. Riat, D. Rios, G. R. S. Ritchie, M. Ruffier, M. Schuster, D. Sobral, G. Spudich, Y. A. Tang, S. Trevanion, J. Vandrovcova, A. J. Vilella, S. White, S. P. Wilder, A. Zadissa, J. Zamora, B. L. Aken, E. Birney, F. Cunningham, I. Dunham, R. Durbin, X. M. Fernández-Suarez, J. Herrero, T. J. P. Hubbard, A. Parker, G. Proctor, J. Vogel, S. M. J. Searle, Ensembl 2011. Nucleic Acids Res. 39, D800-D806 (2011). doi:10.1093/nar/gkq1064 Medline

155. R. N. Gutenkunst, R. D. Hernandez, S. H. Williamson, C. D. Bustamante, Inferring the joint demographic history of multiple populations from multidimensional SNP frequency data. PLOS Genet. 5, e1000695 (2009). doi:10.1371/journal.pgen.1000695 Medline

156. A. C. Davison, D. V. Hinkley, Bootstrap Methods and Their Application (Cambridge Univ. Press, 2013).

157. P. W. Messer, SLiM: Simulating evolution with selection and linkage. Genetics 194, 1037-1039 (2013). doi:10.1534/genetics.113.152181 Medline

158. A. Marom, J. S. O. McCullagh, T. F. G. Higham, R. E. M. Hedges, Hydroxyproline dating: Experiments on the ${ }^{14} \mathrm{C}$ analysis of contaminated and low-collagen bones. Radiocarbon 55, 698-708 (2013). doi:10.1017/S0033822200057854

159. T. B. Coplen, Reporting of stable hydrogen, carbon, and oxygen isotopic abundances. Geothermics 24. 707-712 (1995). doi:10.1016/03756505(95)00024-0

\section{ACKNOWLEDGMENTS}

P.R.N.'s research was funded by the FP7 Marie Curie program (CIG Nr 322261), Isaac Newton Trust, Leakey Foundation, and McDonald Grants and Awards Fund. DMB's research was funded by the H2O20 Marie Skłodowska Curie program (EF Nr 656325). ID and VS were supported by a Swiss NSF grant 31003A-143393 to LE. GeoGenetics is supported by the Lundbeck Foundation and the Danish National Research Foundation (Grant DNRF94), and KU2016. V.V.P., E.Y.P., and P.A.N. are supported by Russian Science Foundation project N 16-18-10265RNF. AM was supported by ERC Consolidator Grant 647787-LocalAdaptation. Thanks to L. Moreau, A. Sinistyn for helpful discussions. We thank the staff of the Danish National High-Throughput DNA Sequencing Centre and the laboratory technicians of the Centre for GeoGenetics for technical assistance. Genomic data are available for download at the ENA (European Nucleotide Archive) with the accession number PRJEB22592 


\section{SUPPLEMENTARY MATERIALS}

www.sciencemag.org/cgi/content/full/science.aao1807/DC1

Supplementary Text

Figs. S1 to S37

Tables S1 to S29

References (26-159)

22 June 2017; accepted 25 September 2017

Published online 5 October 2017

10.1126/science.aao1807 
A

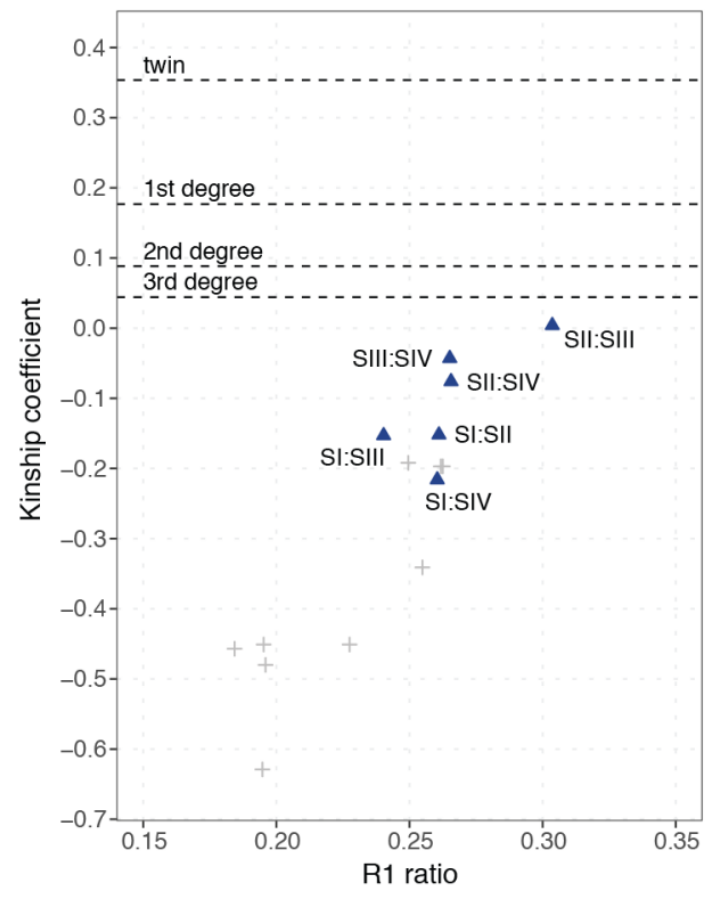

B

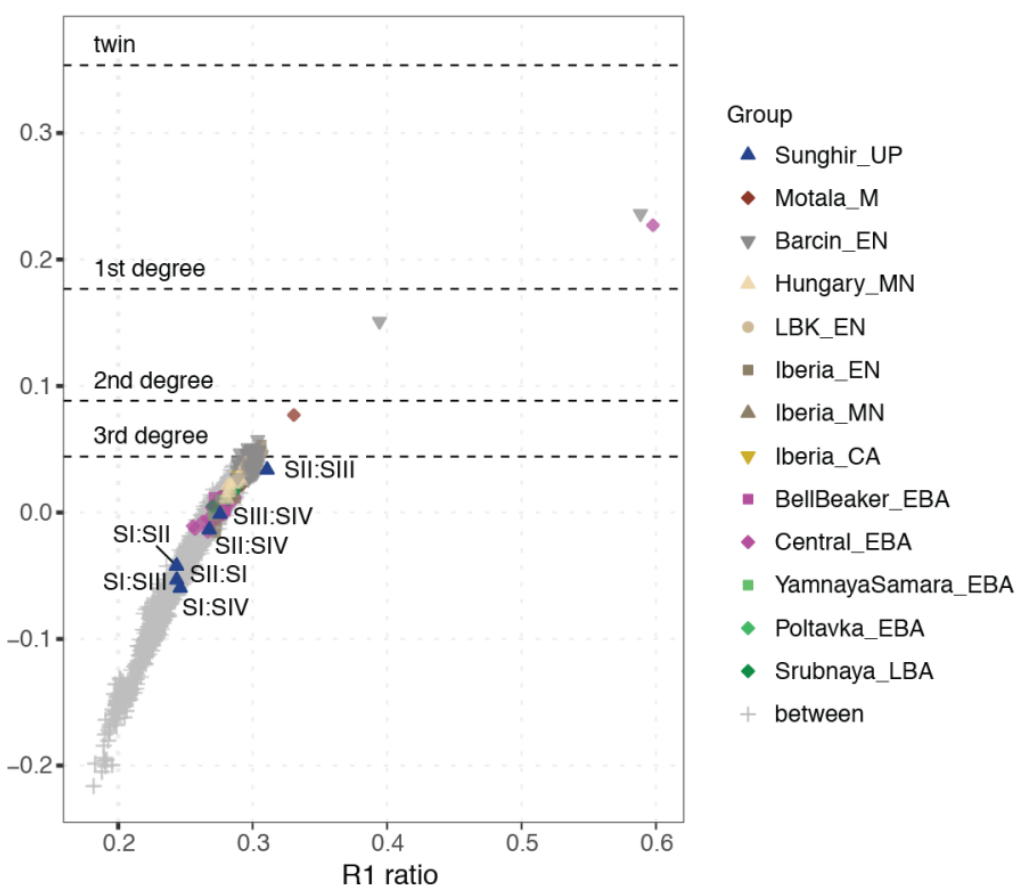

Fig. 1. Relatedness among ancient Eurasians. Kinship coefficients and R1 ratios inferred from IBS ratios for (A) Pairs of UP individuals, using 1000 Genomes Phase 3 SNP sites. (B) Pairs of ancient Eurasians, using $1240 \mathrm{~K}$ capture SNP sites. Within-group pairs of Sunghir are highlighted. 
A

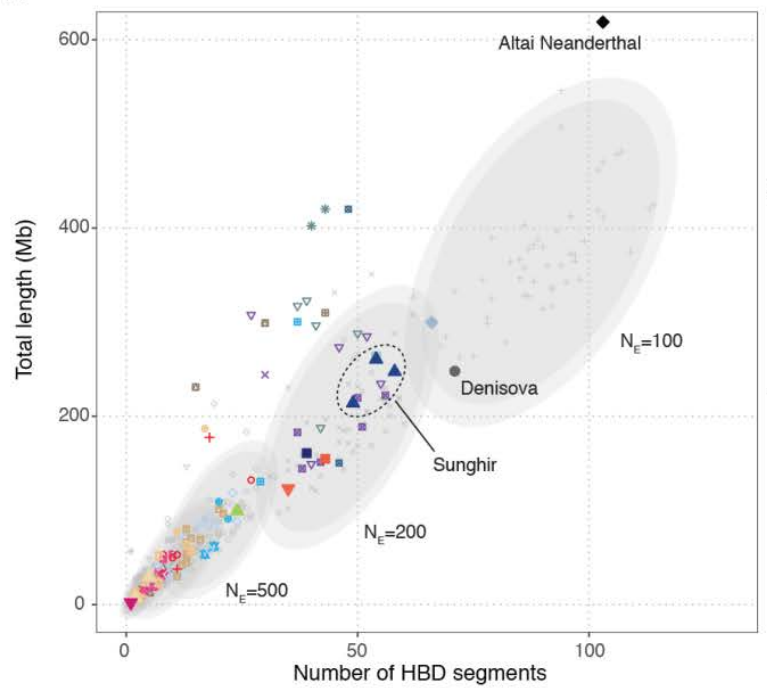

B

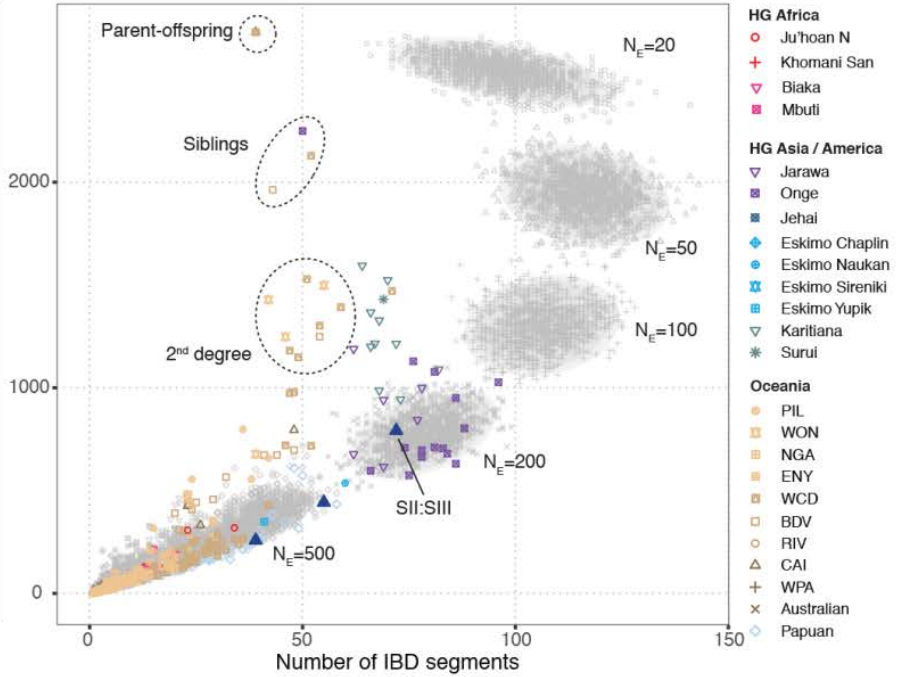

C

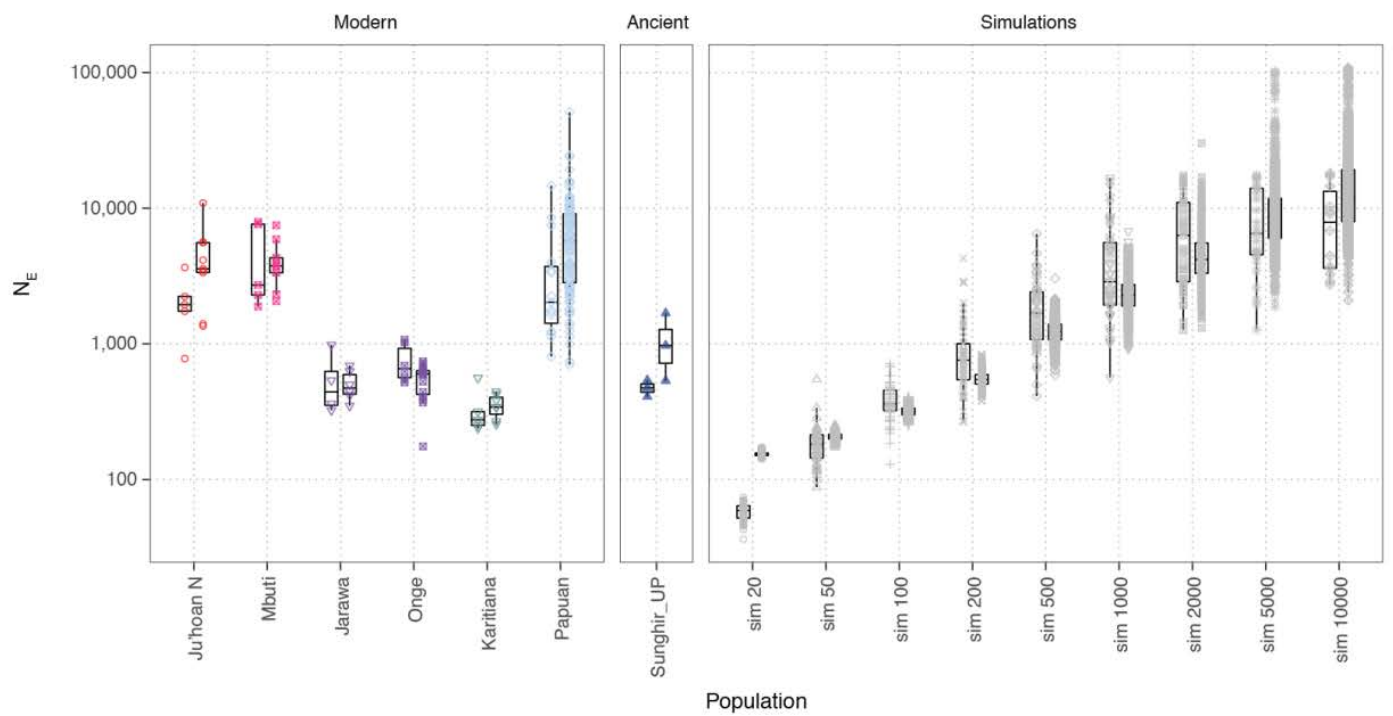

Fig. 2. Identity-by-descent and recent effective population sizes. (A and B) Distributions of the number and total length of HBD and IBD segments in modern, ancient and archaic humans (Altai Neandertal and Denisovan). Ellipses indicate $95^{\text {th }}$ and $99^{\text {th }}$ percentile of the distributions inferred from simulated data of various $N_{E}$ values. Individuals with previously described close relatedness and their degree are indicated in (B). (C) Distributions of inferred recent effective population sizes for modern and ancient HGs with a minimum of three individuals, as well as simulated datasets of randomly mating populations with a range of $\mathrm{N}_{\mathrm{E}}$ values. For each population, sizes inferred from HBD and IBD tracts are on the left and right side, respectively. 
A

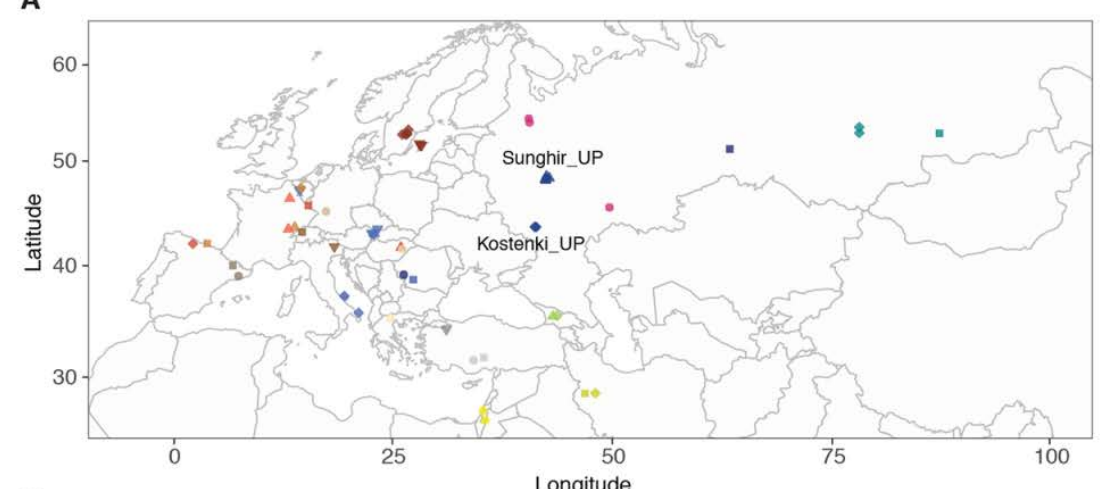

B

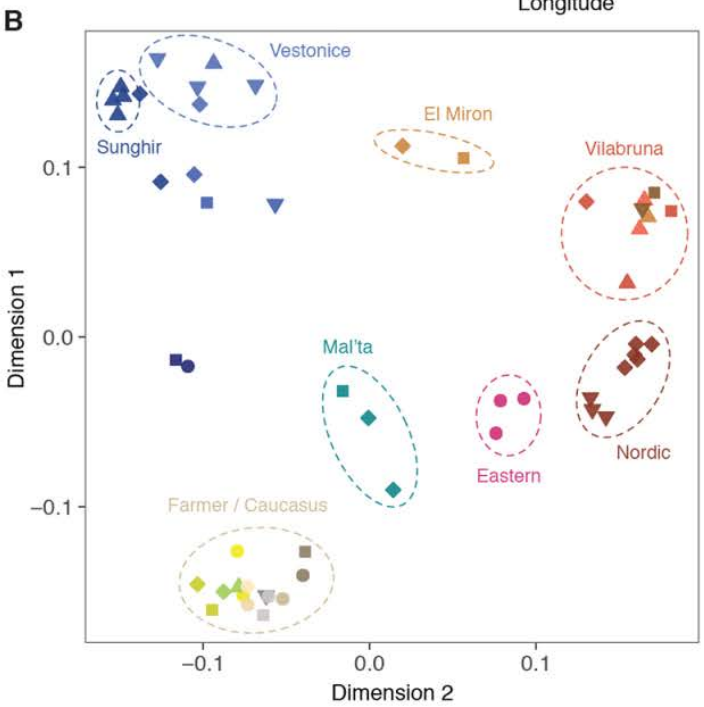

C

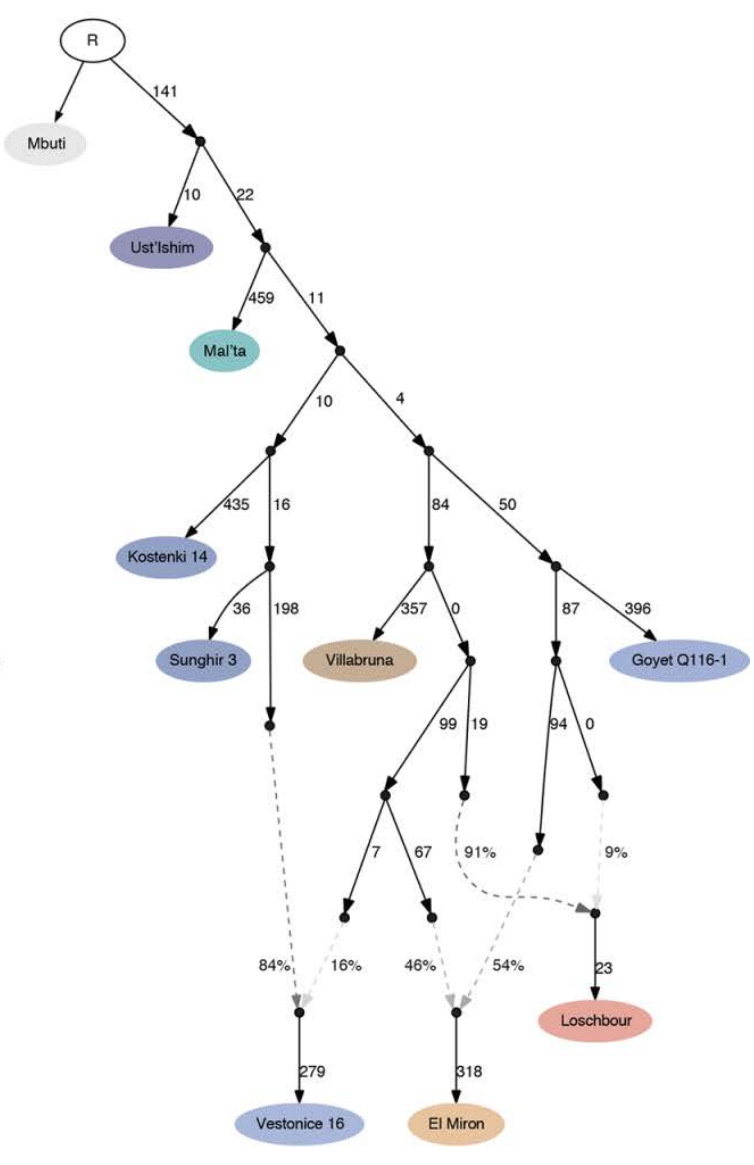

Fig. 3. Genetic affinities of the Sunghir individuals. (A) Geographic locations of ancient Eurasian individuals used in the analysis. (B) Multi-dimensional scaling of ancient individuals based on pairwise shared genetic drift (outgroup $f 3$ statistics $f_{3}\left(\right.$ Mbuti; Ancient $_{1}$, Ancient 2$)$ ). (C) Admixture graph showing the best fit of Sunghir with other early Eurasians. 
a

Sunghir demography

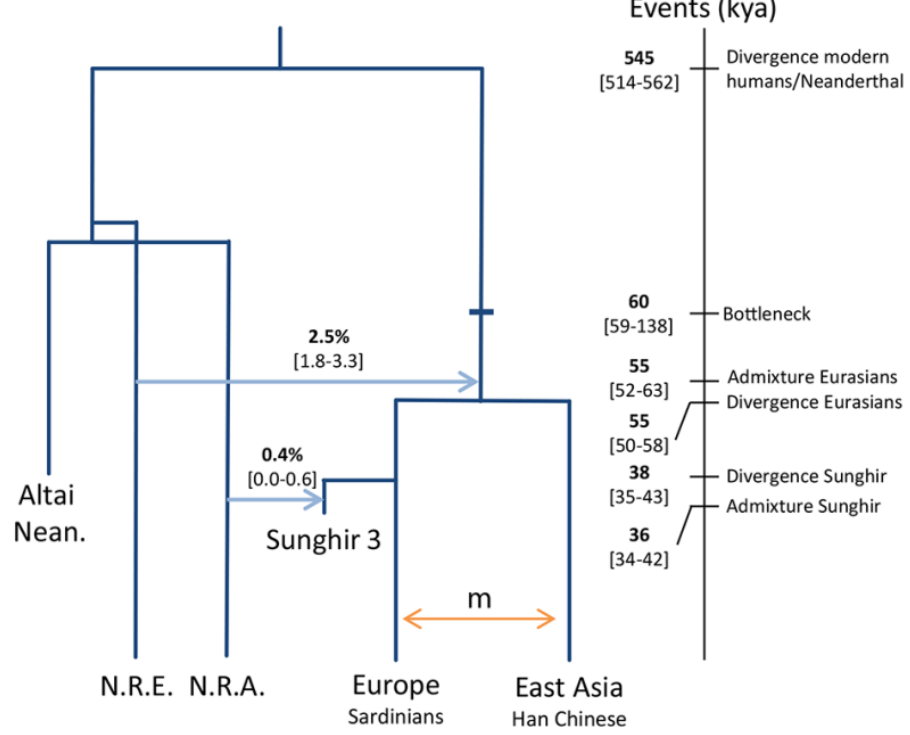

b Ust'-Ishim demography

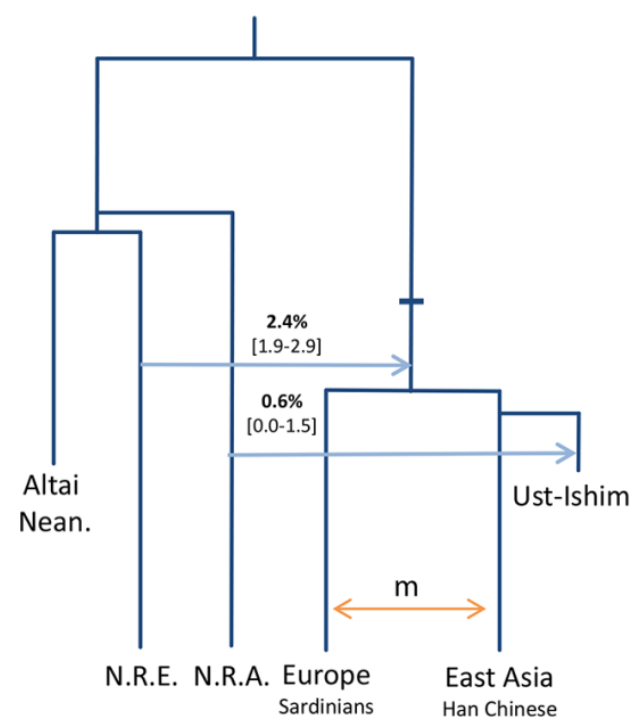

Time Events (kya)

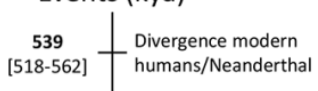

Fig. 4. Modelling of early Eurasian population history. Best-fit demographic models for early Eurasian admixture including (A) SIII and (B) Ust'-Ishim. Point estimates are shown in bold, and 95\% confidence intervals are shown within square brackets. Times of divergence in years are obtained assuming a generation time of 29 years and a mutation rate of $1.25 \mathrm{e}-8 / \mathrm{gen} / \mathrm{site}$. N.R.E.: Altai Neanderthal-related ghost population contributing to Eurasians. N.R.A.: Altai Neanderthal-related ghost population contributing to ancient modern humans. Divergence of SIII from proto-Europeans was supported in 100/100 bootstrap replicates, whereas divergence of Ust'-Ishim from protoAsians was supported in 99/100 bootstrap replicates. 


\section{Science}

\section{Ancient genomes show social and reproductive behavior of early Upper Paleolithic foragers}

Martin Sikora, Andaine Seguin-Orlando, Vitor C. Sousa, Anders Albrechtsen, Thorfinn Korneliussen, Amy Ko, Simon Rasmussen, Isabelle Dupanloup, Philip R. Nigst, Marjolein D. Bosch, Gabriel Renaud, Morten E. Allentoft, Ashot Margaryan, Sergey V. Vasilyev, Elizaveta V. Veselovskaya, Svetlana B. Borutskaya, Thibaut Deviese, Dan Comeskey, Tom Higham, Andrea Manica, Robert Foley, David J. Meltzer, Rasmus Nielsen, Laurent Excoffier, Marta Mirazon Lahr, Ludovic Orlando and Eske Willerslev

published online October 5, 2017

ARTICLE TOOLS

SUPPLEMENTARY

MATERIALS

RELATED
CONTENT

REFERENCES

PERMISSIONS http://science.sciencemag.org/content/early/2017/10/04/science.aao1807

http://science.sciencemag.org/content/suppl/2017/10/04/science.aao1807.DC1

http://science.sciencemag.org/content/sci/358/6363/586.full http://science.sciencemag.org/content/sci/358/6363/655.full

This article cites 131 articles, 26 of which you can access for free $\mathrm{http} / / /$ science.sciencemag.org/content/early/2017/10/04/science.aao1807\#BIBL

http://www.sciencemag.org/help/reprints-and-permissions

Use of this article is subject to the Terms of Service

Science (print ISSN 0036-8075; online ISSN 1095-9203) is published by the American Association for the Advancement of Science, 1200 New York Avenue NW, Washington, DC 20005. 2017 (C) The Authors, some rights reserved; exclusive licensee American Association for the Advancement of Science. No claim to original U.S. Government Works. The title Science is a registered trademark of AAAS. 